\title{
EM-mosaic detects mosaic point mutations that contribute to congenital heart disease
}

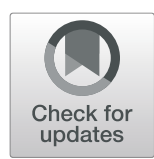

\author{
Alexander Hsieh ${ }^{1 \dagger}$, Sarah U. Morton ${ }^{2,3 \dagger}$, Jon A. L. Willcox ${ }^{3 \dagger}$, Joshua M. Gorham ${ }^{3}$, Angela C. Tai ${ }^{3}$, Hongjian Qi ${ }^{1}$, \\ Steven DePalma ${ }^{3}$, David McKean ${ }^{3}$, Emily Griffin ${ }^{1}$, Kathryn B. Manheimer ${ }^{4}$, Daniel Bernstein ${ }^{5}$, Richard W. Kim ${ }^{6}$, \\ Jane W. Newburger ${ }^{2}$, George A. Porter $\mathrm{Jr}^{7}$, Deepak Srivastava ${ }^{8}$, Martin Tristani-Firouzi ${ }^{9}$, Martina Brueckner ${ }^{10}$, \\ Richard P. Lifton ${ }^{11}$, Elizabeth Goldmuntz ${ }^{12}$, Bruce D. Gelb4, Wendy K. Chung ${ }^{1 \dagger}$, Christine E. Seidman ${ }^{3,13,14 \dagger}$, \\ J. G. Seidman ${ }^{3 \dagger}$ and Yufeng Shen ${ }^{1 *+}$
}

\begin{abstract}
Background: The contribution of somatic mosaicism, or genetic mutations arising after oocyte fertilization, to congenital heart disease (CHD) is not well understood. Further, the relationship between mosaicism in blood and cardiovascular tissue has not been determined.

Methods: We developed a new computational method, EM-mosaic (Expectation-Maximization-based detection of mosaicism), to analyze mosaicism in exome sequences derived primarily from blood DNA of 2530 CHD probandparent trios. To optimize this method, we measured mosaic detection power as a function of sequencing depth. In parallel, we analyzed our cohort using MosaicHunter, a Bayesian genotyping algorithm-based mosaic detection tool, and compared the two methods. The accuracy of these mosaic variant detection algorithms was assessed using an independent resequencing method. We then applied both methods to detect mosaicism in cardiac tissue-derived exome sequences of 66 participants for which matched blood and heart tissue was available.
\end{abstract}

Results: EM-mosaic detected 326 mosaic mutations in blood and/or cardiac tissue DNA. Of the 309 detected in blood DNA, 85/97 (88\%) tested were independently confirmed, while 7/17 (41\%) candidates of 17 detected in cardiac tissue were confirmed. MosaicHunter detected an additional 64 mosaics, of which 23/46 (50\%) among 58 candidates from blood and 4/6 (67\%) of 6 candidates from cardiac tissue confirmed. Twenty-five mosaic variants altered CHD-risk genes, affecting 1\% of our cohort. Of these 25, 22/22 candidates tested were confirmed. Variants predicted as damaging had higher variant allele fraction than benign variants, suggesting a role in CHD. The estimated true frequency of mosaic variants above $10 \%$ mosaicism was 0.14 /person in blood and $0.21 /$ person in cardiac tissue. Analysis of 66 individuals with matched cardiac tissue available revealed both tissue-specific and shared mosaicism, with shared mosaics generally having higher allele fraction.

(Continued on next page)

\footnotetext{
*Correspondence: ys2411@cumc.columbia.edu

${ }^{\dagger}$ Alexander Hsieh, Sarah U. Morton, Jon A. L. Willcox, Wendy K. Chung,

Christine E. Seidman, J. G. Seidman and Yufeng Shen contributed equally to this work.

${ }^{1}$ Columbia University Medical Center, 1130 St Nicholas Ave, New York, NY

10032, USA

Full list of author information is available at the end of the article
}

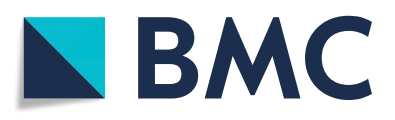

(c) The Author(s). 2020 Open Access This article is licensed under a Creative Commons Attribution 4.0 International License, which permits use, sharing, adaptation, distribution and reproduction in any medium or format, as long as you give appropriate credit to the original author(s) and the source, provide a link to the Creative Commons licence, and indicate if changes were made. The images or other third party material in this article are included in the article's Creative Commons licence, unless indicated otherwise in a credit line to the material. If material is not included in the article's Creative Commons licence and your intended use is not permitted by statutory regulation or exceeds the permitted use, you will need to obtain permission directly from the copyright holder. To view a copy of this licence, visit http://creativecommons.org/licenses/by/4.0/ The Creative Commons Public Domain Dedication waiver (http://creativecommons.org/publicdomain/zero/1.0/) applies to the data made available in this article, unless otherwise stated in a credit line to the data. 
(Continued from previous page)

Conclusions: We estimate that $\sim 1 \%$ of CHD probands have a mosaic variant detectable in blood that could contribute to cardiac malformations, particularly those damaging variants with relatively higher allele fraction. Although blood is a readily available DNA source, cardiac tissues analyzed contributed $\sim 5 \%$ of somatic mosaic variants identified, indicating the value of tissue mosaicism analyses.

Keywords: Mosaic, Somatic, Congenital heart disease, Exome sequencing

\section{Background}

Mosaicism results from somatic mutations that arise post-zygotically in an early embryonic cell, resulting in two or more cell populations with distinct genotypes in the developing embryo [4]. The developmental status of the early embryonic cell at the time of mutagenesis determines the proportion of variant-carrying cells and the tissue distribution of these cells in the post-natal child [1]. While germline variants have a variant allele frequency (VAF) of 0.5 , somatic mosaic variants have a significantly lower VAF.

Post-zygotic mosaic mutations have been implicated in several diseases including non-malignant developmental disorders such as overgrowth syndromes [47, 55, 64], structural brain malformations [41, 49, 64, 69], epilepsy [76], and autism spectrum disorder [16, 23, 45, 54]. Recent analyses also identified mosaic variants in a cohort of patients with congenital heart disease (CHD) [57], but the prevalence of these was far less than germline variants (CHD) [34, 42, 86, 87].

Assessment of the frequency of mosaicism in human disease is confounded by technical issues, including differences in sequencing depth, DNA sources, and variant assessment pipelines. Low levels of mosaicism can escape the detection threshold of traditional sequencing methods with standard read depths, while post-zygotic mutations with a higher percentage of affected cells are difficult to discriminate from germline de novo mutations [1]. All of these issues can lead to substantially different conclusions. For example, analyses of mosaicism in autism spectrum disorder was recently assessed from whole exome sequence (WES) data from whole blood DNA from 2506 families (proband, parents and unaffected sibling; trios and quads) in the Simons Simplex Collection (SSC) [21]. The primary sequence data were analyzed by three groups; one that identified a protein-coding somatic mosaic variant rate of 0.074 per individual [23], another that found a mosaic rate of 0.059 per individual [54], and a third group that reported a mosaic rate of 0.125 per individual [45]. This disparity both highlights algorithm-specific differences and suggests the need for a more systematic mosaic mutation detection method that accounts for datasetspecific confounding factors.

By contrast, analyses of affected tissues can improve the sensitivity and specificity of detection of somatic mosaicism. In cancer, methods to detect these events, such as MuTect [7], compare tumor and benign tissues from the same patient. Mosaicism has also been demonstrated from the analyses of unpaired samples with cancer and other pathologies [37, 73, 77] by the demonstration of variants in affected tissues that are absent from blood-derived DNA [59, 78]. With access to cardiac tissues from patients with $\mathrm{CHD}$ obtained during surgical repair, we hypothesized that analyses of mosaicism in cardiac tissue might improve insights into the causes of this common congenital anomaly. As many cardiomyocyte lineages share a mesodermic origin with blood cells but exit the cell cycle during embryogenesis, we also sought to determine if mosaicism in the heart exhibited distinct patterns of mosaicism with regard to variant frequency and allele fractions.

In this study, we developed a computational method, EM-mosaic (Expectation-Maximization-based detection of Mosaicism) [35], to detect mosaic single-nucleotide variants (SNVs) using WES of proband and parent DNA. To optimize this method, we measured mosaic detection power as a function of sequencing depth. We applied both EM-mosaic and MosaicHunter [37] to investigate mosaicism in $2530 \mathrm{CHD}$ proband-parent trios from the Pediatric Cardiac Genomics Consortium (PCGC) [42], using exome sequences derived from blood-derived DNA, and compared the two methods. We detected predicted deleterious mosaic mutations in genes involved in known biological processes relevant to $\mathrm{CHD}$ or developmental disorders in $1 \%$ of probands. The accuracy of these mosaic variant detection algorithms was assessed using an independent resequencing method. We found that among high-confidence mosaic mutations in CHD-relevant genes, likely damaging variants tended to have higher VAF than likely benign variants.

In parallel, we assessed mosaicism by EM-mosaic and MosaicHunter in 70 discarded tissues from several heart regions obtained from 66 probands who underwent cardiac surgical repairs. While VAF varied significantly ( $>3$ fold) between blood and cardiovascular tissue at about $60 \%$ of sites, in general mosaic variants with high (>15\%) VAF were more likely shared between blood and cardiac tissue than variants with lower VAF. 


\section{Methods}

\section{Samples and sequencing data}

We analyzed WES data from 2530 congenital heart disease (CHD) proband-parents trio families who were recruited as part of the Pediatric Cardiac Genomics Consortium (PCGC) study [34, 42]. Genomic DNA from venous blood or saliva was captured using Nimblegen v.2 exome capture reagent (Roche) or Nimblegen SeqCap EZ MedExome Target Enrichment Kit (Roche) followed by Illumina DNA sequencing (paired-end, 2x75bp) [42, 86]. Of 2530 participant DNA samples, 2453 were from blood and 77 were from saliva. Genomic DNA from 70 surgically discarded cardiovascular tissue samples (2-10 mg) was isolated using DNeasy Blood \& Tissue Kit (QIAgen), then captured using xGen Exome Research Panel v1.0 reagent (IDT) followed by Illumina DNA sequencing (paired-end, $2 \times 75 \mathrm{bp}$ ). Sequence reads were mapped to the hg19 human reference genome with BWA-MEM [51], and BAM files were further processed following GATK Best Practices [81], which included duplication marking, indel realignment, and base quality recalibration steps. Blood and saliva samples had sample average depth $60 \times$ and cardiovascular tissue samples had sample average depth $160 \times$. A summary of germline and mosaic variants called from blood and saliva DNA can be found in Additional file 1: Table S15.

\section{De novo variant calling and annotation}

We processed our sample BAMs and called variants on a per-trio basis using SAMtools (v1.3.1-42) and BCFtools (v1.3.1-174) [52]. Pileups were generated using samtools "mpileup" command with mapQ 20 and baseQ 13 to minimize the effect of poorly mapped reads on variant allele fraction, followed by bcftools "call" using a cutoff of 1.1 for the posterior probability of the homozygous reference genotype parameter ( $-p)$ to capture additional sites with variant allele fraction suggestive of postzygotic origin that would otherwise be excluded under the default threshold of 0.01 . To identify de novo mutations from trio VCF files, we selected sites with (i) a minimum of 6 reads supporting the alternate allele in the proband and (ii) for both parents, a minimum depth of 10 reads and 0 alternate allele read support. Variants were then annotated using ANNOVAR (v2017-07-17) [83] to include information from refGene [63], gnomAD (March 2017) [44], 1000 Genomes (August 2015) [2], ExAC [43], genomicSuperDups [32], CADD (v1.3) [68], COSMIC (v70) [79], and dbSNP (v147) [56] databases, as well as pathogenicity predictions from a variety of established methods included as part of the dbNSFP (v3.0a) database or generated in-house (MCAP, REVEL, MVP, MPC). We used REVEL [38] to evaluate missense variant functional consequence, using the recommended threshold of 0.5 corresponding to sensitivity of 0.754 and specificity of 0.891 . We used spliceAI [39] to predict the variant functional impact on splicing using the delta score thresholds of 0.2 for likely pathogenic (high recall), 0.5 for pathogenic (recommended), and 0.8 for pathogenic (high precision). We considered sites predicted to be loss of function (LoF) (stopgain, stoploss, frameshift indels, splice-site), deleterious missense (Dmis; nonsynonymous SNV with REVEL>0.5), or splice-damaging (benign missense or synonymous SNV with delta score $>0.5$ ) to be damaging and likely disease causing. We considered sites predicted to be synonymous (delta score $\leq 0.5$ ) or benign missense (Bmis; nonsynonymous SNV with REVEL $\leq 0.5$ and delta score $\leq 0.5$ ) to be nondamaging.

\section{Pre-processing and QC}

To reduce the number of low VAF technical artifacts introduced by our variant calling approach, we preprocessed our variants using a variety of filters (Fig. 1). We first excluded indels from further analysis, as their downstream model parameter estimates were less stable than those of SNVs. We then filtered our variant call set for rare heterozygous coding mutations (minor allele frequency (MAF) $\leq 10^{-4}$ across all populations represented in gnomAD and ExAC databases). To account for regions in the reference genome that are likely to affect read depth estimates, we removed variant sites found in regions of non-unique mappability (score $<1$; $300 \mathrm{bp}$ ), likely segmental duplication (score $>0.95$ ), and known low-complexity [53]. We then excluded sites located in MUC and HLA genes and imposed a maximum variant read depth threshold of 500. We used SAMtools PV4 to exclude sites with evidence of technical issues using a cutoff of $1 \mathrm{e}-3$ for baseQ bias and tail distance bias and a cutoff of $1 \mathrm{e}-6$ for mapQ bias. To account for potential strand bias, we used an in-house script to flag sites that have either (1) 0 alternate allele read support on either the forward or reverse strand or (2) $P<1 \mathrm{e}-3$ and (odds ratio $(\mathrm{OR})<0.33$ or $\mathrm{OR}>3$ ) when applying a two-sided Fisher's exact test to compare proportions of reference and alternate allele read counts on the forward and reverse strands. We also excluded sites with cohort frequency $>1 \%$, as well as sites belonging to outlier samples (with abnormally high de novo SNV (dnSNV) counts, cutoff $=8$ ) and variant clusters (defined as sites with neighboring SNVs within $10 \mathrm{bp}$ ). Finally, we applied an false discovery rate (FDR)-based minimum $N_{\text {alt }}$ filtering step (Additional file 2: Figure S5) to control for false positives caused purely by sequencing errors.

\section{IGV visualization of low allele fraction de novo SNVs}

To reduce the impact of technical artifacts on model parameter estimation, we manually inspected de novo SNVs with $\mathrm{VAF}<0.3 \quad(n=558)$ using Integrative Genomics 


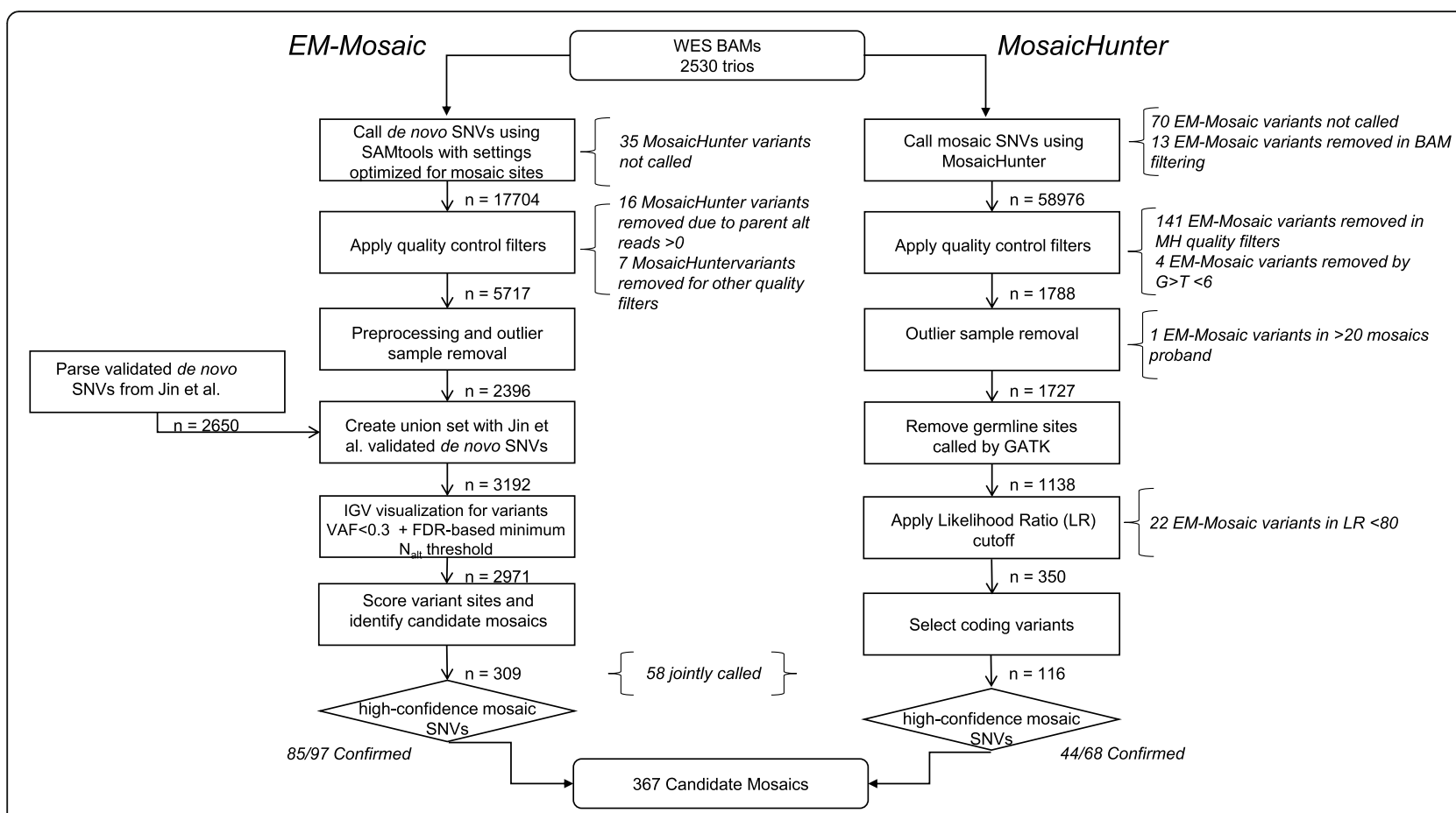

Fig. 1 Mosaic detection pipeline flowchart. Summary of approach for detecting mosaic variants in our cohort of $n=2530 \mathrm{CHD}$ proband-parent trios. EM-mosaic flowchart (left). We first processed our SAMtools de novo calls using our upstream filters ( $n=2396$ sites passing all filters). We then applied the same upstream filters to the published dnSNVs from Jin et al. ( $n=2650$ sites passing all filters) before finally taking the union of these two call sets $(n=3192)$. High-confidence mosaics $(n=309)$ were defined as mosaics passing IGV inspection and having posterior odds $>10$. Italicized text indicates which filters removed candidate mosaic variants called by MosaicHunter but not by EM-mosaic. MosaicHunter workflow (right). Quality control filters excluded any sites that were (1) present in ExAC (2) G>T with $N_{\text {alt }}<10$ (3) parent $N_{\text {alt }}>2$. Outliers were defined as probands carrying more than 20 mosaics, or non-unique sites. We also removed sites called as germline by GATK Haplotype Caller. Highconfidence mosaics $(n=116)$ were defined as having a likelihood ratio $>80$ and affecting coding regions excluding MUC/HLA genes. Italicized text indicates which filters removed variants called by EM-mosaic but not by MosaicHunter

Viewer (v2.3.97) [70] to visualize the local read pileup at each variant across all members of a given trio family. We focused on the allele fraction range 0.0-0.3 since this range is enriched for technical artifacts that could potentially impact downstream parameter estimation. Sites were filtered out if (1) there are inconsistent mismatches in the reads supporting the mosaic allele, (2) the site overlaps or is adjacent to an indel, (3) the site has low MAPQ or is not primary alignment, (4) there is evidence of technical bias (strand, read position, tail distance), or (5) the site is mainly supported by soft-clipped reads.

\section{Expectation-maximization to estimate prior mosaic fraction and control FDR}

Current estimates for the fraction of de novo events occurring post-zygotically are unstable due to differences in study factors such as variant calling methods, average sequencing depth, and paternal ages. In order to use this fraction as a prior probability in our posterior odds and false discovery calculations, we reason that this value must be estimated from the data itself. We used an expectation-maximization algorithm to jointly estimate the fraction of mosaics among apparent de novo mutations and to calculate a per-site likelihood ratio score. This initial mosaic fraction estimate gives us a prior probability of mosaicism, independent of sequencing depth or variant caller, and allows us to calculate for each variant the posterior odds that a given site is mosaic rather than germline. To control for false discovery among our predicted mosaic candidates, we chose a posterior odds threshold of 10 to restrict FDR to $9.1 \%$.

\section{Mosaic mutation detection model}

To distinguish variant sites that show evidence of mosaicism from germline heterozygous sites, we modeled the number of reads supporting the variant allele $\left(N_{\text {alt }}\right)$ as a function of the total site depth $(N)$. In the typical case, $N_{\text {alt }}$ follows a binomial model with parameters $N=$ site depth and $P=$ mean VAF. However, we observed notable overdispersion in the distribution of variant allele fraction compared to the expectations under this binomial model (Additional file 2: Figure S4). To account for this overdispersion, we instead modeled $N_{\text {alt }}$ using a betabinomial distribution $[33,66]$. We estimated an 
overdispersion parameter $\theta$ for our model as follows: for site depth values $N$ in the range 1 to 500 , we (1) bin variants by identifying all sites with depth $N$, (2) calculate a maximum-likelihood estimate $\theta$ value using $N$ and all $N_{\text {alt }}$ values for variants in a given bin, and (3) estimate a global $\theta$ value by taking the average of $\theta$ values across all bins, weighted by the number of variants in each bin. We then used $\theta$ in our expectation-maximization approach to jointly estimate prior mosaic fraction and to calculate per-site likelihood ratios.

To calculate the posterior odds that a given variant arose post-zygotically, we first calculated a likelihood ratio (LR) of two models: $\mathrm{M}_{0}$ : germline heterozygous variant, and $\mathrm{M}_{1}$ : mosaic variant. Under our null model $\mathrm{M}_{0}$, we calculated the probability of observing $N_{\text {alt }}$ from a beta-binomial distribution with site depth $N$, observed mean germline VAF $P$, and overdispersion parameter $\theta$. Under our alternate model $\mathrm{M}_{1}$, we calculated the probability of observing $N_{\text {alt }}$ from a beta-binomial distribution with site depth $N$, observed site VAF $P=N_{\text {alt }} / N$, and overdispersion parameter $\theta$. Finally, for each variant, we calculated LR by using the ratio of probabilities under each model and posterior odds by multiplying LR by our E-M estimated prior mosaic fraction estimate. We defined mosaic sites as those with posterior odds greater than 10 (corresponding to 9.1\% FDR). We used posterior odds in this context to be able to control for false discovery, but we output similarly valid $P$ value and likelihood ratio scores for each de novo SNV.

\section{Mutation confirmation by MiSeq amplicon sequencing} Chromosome coordinates were expanded $500 \mathrm{bp}$ upstream and downstream of the candidate mosaic variants in the UCSC Genome Browser. Primer 3 Plus software was used to design forward and reverse primers to generate $150-300$-bp amplimers containing the candidate site. PCR reactions consisting of genomic DNA, primers, and Phusion polymerase were amplified by thermal cycling and purified with AMPure XP beads. The purified PCR product was quantified, and $0.5-1.0 \mathrm{ng}$ of product was used to construct Nextera XT libraries according to the protocol published by Illumina. Libraries were purified using AMPure XP beads, and final libraries were quantified and pooled to undergo sequencing through Illumina MiSeq.

We experimentally tested for the presence our predicted post-zygotic sites in the original blood DNA and cardiovascular tissue DNA samples using Illumina MiSeq Amplicon sequencing. The Amplicon Deep Sequencing workflow, optimized for the detection of somatic mutations in tumor samples, offers ultra-high sequencing depth $(>1000 \times)$ that gives us the resolution to confirm low VAF variants, to accurately estimate site VAF, and to distinguish true variant calls from technical artifacts. Mosaic candidates were considered validated if the variant allele matched the MiSeq call and both the mosaic VAF and MiSeq VAF indicated post-zygotic origin $(\mathrm{VAF}<0.45)$.

Mosaic candidates were selected for confirmation on the basis of VAF, plausible involvement in CHD (based on predicted pathogenicity and HHE status), and detection method (Additional file 1: Table S11; Additional file 1: Table S12). We sampled mosaics from both ends of the VAF spectrum to evaluate our ability to distinguish high VAF mosaics (VAF $>0.2 ; n=29$ ) from germline variants and to distinguish low VAF mosaics (VAF $<=0.1 ; n=52)$ from technical artifacts. Confirmation rate across different VAF bins is shown in Additional file 2: Figure S12. We also selected for confirmation mosaics detected uniquely by either EMmosaic or MosaicHunter, for the sake of method comparison (Table 1).

To examine a potential source of bias in our candidate selection process, we compared the posterior odds distribution of selected candidate mosaics $(n=97)$ against those not chosen $(n=212)$. We found that our tested

Table 1 Mosaic variant detection by EM-Mosaic and MosaicHunter and validated by PCR product sequencing

\begin{tabular}{|c|c|c|c|c|}
\hline & \multirow[t]{2}{*}{ Union } & \multirow[t]{2}{*}{ Shared } & \multicolumn{2}{|l|}{ Unique } \\
\hline & & & EM-Mosaic & MosaicHunter \\
\hline High-confidence mosaic variants* & 332 & 57 & 240 & 35 \\
\hline Mosaic candidates & 367 & 58 & 251 & 58 \\
\hline Mosaic candidate VAF mean (SD) & $0.13(0.06)$ & $0.12(0.05)$ & $0.13(0.06)$ & $0.10(0.05)$ \\
\hline \multicolumn{5}{|l|}{ MiSeq confirmation } \\
\hline Total tested & 143 & 22 & 75 & 46 \\
\hline Mosaic & 108 & 21 & 64 & 23 \\
\hline Germline & 3 & 0 & 3 & 0 \\
\hline No variant & 32 & 1 & 8 & 23 \\
\hline Validation rate & $76 \%$ & $95 \%$ & $85 \%$ & $50 \%$ \\
\hline
\end{tabular}

*Mosaic variants detected from blood DNA of $2530 \mathrm{CHD}$ probands, after excluding sites failing MiSeq confirmation 
candidates had lower posterior odds than untested mosaics $\left(\right.$ mean $_{\text {tested }}=5.382$, mean $_{\text {untested }}=7.050, \log _{10}$-scale; Mann-Whitney $U P=0.002$ ) (Figure 13), suggesting that our validation rate is not buoyed by testing variants with the strongest evidence of mosaicism. For method development purposes, we intentionally focused on mosaics with lower posterior odds as these fall in the VAF range for which it is most difficult to distinguish germline from mosaic.

\section{Investigating the relationship between VAF and pathogenicity}

We hypothesized that mosaic contribution to disease is positively correlated with cellular percentage and by extension mutational timing. Here, we used variant allele fraction as a proxy for cellular percentage. We grouped mosaics into likely damaging and likely benign and compared the distribution of allele fraction in CHD-related genes. We defined likely damaging variants as (a) likely gene-disrupting (LOF) variants (including premature stopgain, frameshifting, and variants located in canonical splice sites), (b) missense variants predicted to be damaging by REVEL [38] (with score $\geq 0.5$ ), or (c) missense variants and synonymous predicted to be splicedamaging by spliceAI (with score $>0.5$ ). One of the main findings from previous CHD studies is that damaging de novo variants in genes highly expressed in the developing heart ("HHE", ranked in the top $25 \%$ by cardiac expression data in mouse at E14.5 $[34,86])$ contribute to non-isolated CHD cases that have additional congenital anomalies or neurodevelopmental disorders. Therefore, we considered the union of HHE genes and known candidate CHD genes [42] as CHD-related genes $(n=4558)$. For mosaics in CHD-related genes and for mosaics in other genes, we used a Mann-Whitney $U$ test to compare the VAF distributions of likely damaging and likely benign groups.

\section{Estimated contribution of mosaicism to CHD}

We identified likely disease-causing mosaic mutations on the basis of predicted pathogenicity and presence in genes involved in biological processes relevant to $\mathrm{CHD}$ or developmental disorders. Each mosaic mutation was annotated with gene-specific information, including heart expression percentile, probability of loss-offunction intolerance (pLI) score [50], whether dysregulation causes CHD in mice [20,72], and gene function (NCBI RefSeq). We focused on HHE genes, genes with high pLI ( $\mathrm{pLI}>0.9$ ), genes that cause CHD phenotypes in mice, and genes involved in key developmental processes such as Wnt, mTOR, and TGF-beta signaling pathways. Then, for each patient, we used the clinical phenotype to further prioritize mosaic mutations most likely contributing to that individual's clinical features.
Detailed mutation annotation and clinical phenotypes for the mosaic carriers described above can be found in Additional file 1: Table S10. We estimate the contribution of mosaicism to CHD as the percentage of individuals carrying likely disease-causing mosaic mutations among all individuals in our CHD cohort.

\section{Results}

High-accuracy detection of mosaic mutations in WES data using EM-mosaic

We analyzed whole exome sequence (WES) data from 2530 CHD proband-parent trios [34, 42] (Additional file 1: Table S1). Among this cohort, 1205 probands had CHD with neurodevelopmental disorders (NDD) and/or extracardiac manifestations (EM), 788 had isolated CHD at the time of enrollment, 539 had undetermined NDD status due to young neonatal age at the time of enrollment, and 9 subjects had incomplete data (Additional file 1: Table S2).

Previous WES analyses [42] identified 1742 germline de novo SNVs carried by 2005 CHD proband-parent trios, including 838 cases with NDD and/or EM, 516 isolated cases, 644 cases of unknown NDD status, and 7 with incomplete data. These de novo variants were identified using the Genome Analysis Toolkit (GATK) pipeline $[14,58]$ assuming a germline diploid model in which the expected VAF is 0.5 . This model has limited sensitivity to detect mosaic mutations for which the fraction of alternative allele reads is significantly below 0.5 , especially because de novo variants with $\mathrm{VAF}<0.2$ were excluded to reduce false discovery.

To efficiently capture mosaic variants with $\mathrm{VAF}<0.4$, we developed a new method (EM-mosaic) to detect mosaic variants in WES sequence of a proband and parents (trios). Potential mosaic variants were identified in WES sequence data using SAMtools mpileup [52] with settings designed to capture sites with VAF between 0.10.4 and merged with the variants found by the GATK pipeline [42] (Fig. 1) to create a union variant set. To reduce the elevated false positive rate inherent in low-VAF calls, we applied a set of empirical filters to remove likely technical artifacts due to sequencing errors associated with repetitive and/or low-complexity sequences. We then manually inspected de novo SNVs with $\mathrm{VAF}<0.3$ $(n=582)$ using IGV and filtered out an additional 188 likely false positives. After preprocessing, outlier removal, and an FDR-based minimum alternate allele read support $\left(N_{\text {alt }}\right)$ filter (Additional file 2: Figure S5), the remaining 2971 de novo SNVs were used as input to our mosaic detection model.

Among the 2971 de novo SNVs, this pipeline identified 309 sites as candidate mosaics based on posterior odds score (Fig. 2a, b; Additional file 1: Table S3), including 50 sites that were previously reported as 


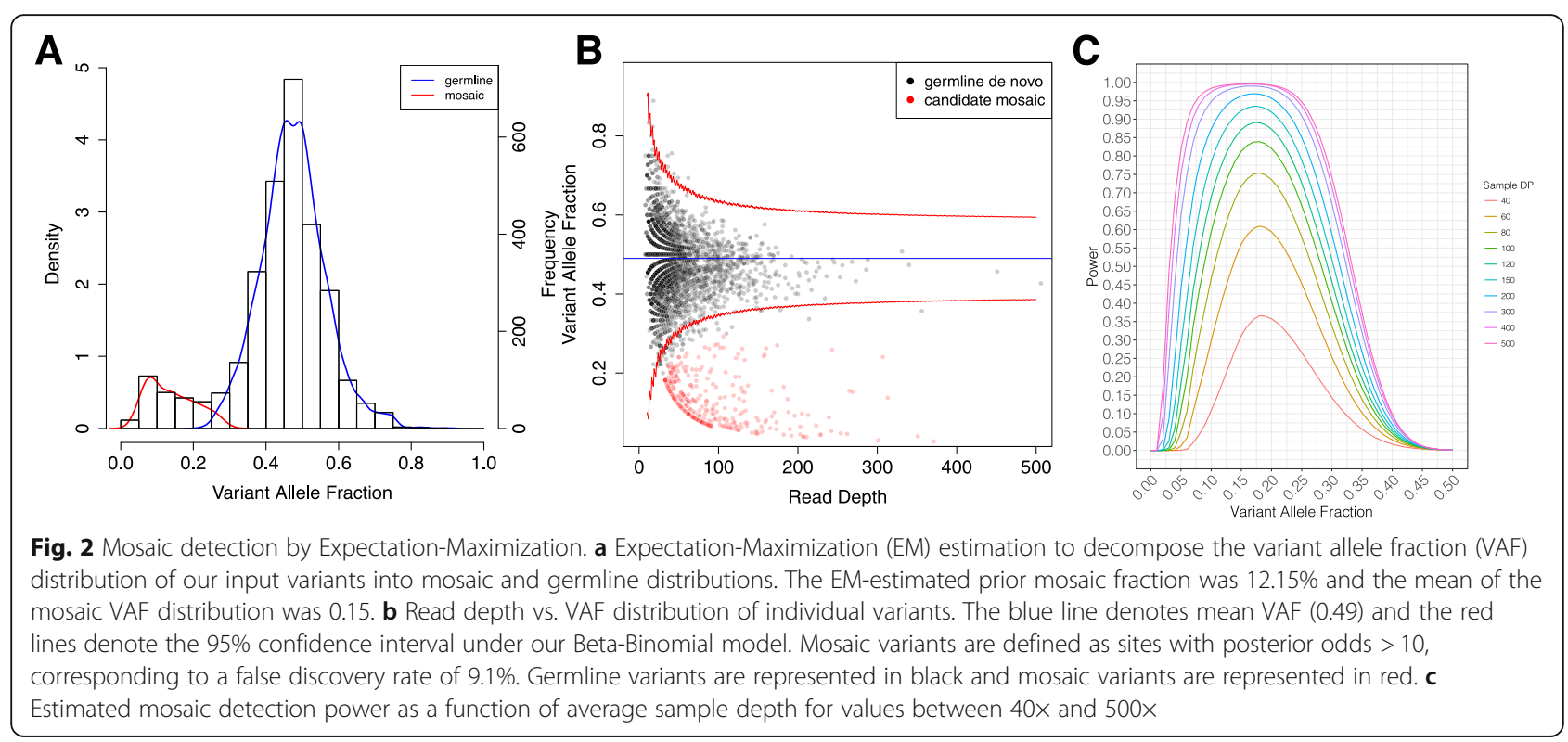

germline de novo variants [42]. Among our 2530 participant DNA samples, 2453 were from blood and 77 were from saliva yielding 300 mosaic candidates and 9 mosaic candidates, respectively; a summary of germline and mosaic variants called from blood and saliva DNA can be found in Additional file 1: Table S15. We also did not find evidence of a relationship between proband age and mosaic rate (Additional file 2: Figure S9) or between parental age (paternal or maternal) and proband mosaic rate (Additional file 1: Table S16; Additional file 2: Figure S10; Additional file 2: S11). Among sites predicted to be germline, 86 sites were identified as having posterior odds below our chosen threshold of 10 but greater than 1 (Additional file 2: Figure S1), including a ZEB2 variant with a posterior odds score of 4.7 that was previously confirmed via ddPCR [57]. Among these 86 variants, we estimate that 53 are likely mosaic and 33 are likely germline (Additional file 2: Figure S1B). We chose not to include these sites since there was insufficient evidence to confidently resolve them individually as mosaic or germline.

\section{Mosaic mutations found in blood-derived DNA with MosaicHunter}

We also employed MosaicHunter, which uses a Bayesian genotyping algorithm with a series of stringent filters (see Supplemental Methods) for discovering mosaic variants using WGS genotype information from trios [37]. Among the $2530 \mathrm{CHD}$ trios, MosaicHunter identified an initial set of 58,976 sites showing evidence of mosaicism, including 214 high-confidence variants located in coding regions (Fig. 1). After applying a minimum likelihood ratio (LR) cutoff of 80 for distinguishing mosaic from germline mutation, and additional heuristic filters
(Supplemental Methods), MosaicHunter identified 116 coding sites (Additional file 1: Table S4) or 0.05 mosaics /individual.

Candidate mosaic variants were compared between the EM-mosaic and MosaicHunter pipelines. Of the mosaic candidates detected by MosaicHunter, 58/116 (50\%) were also identified by EM-mosaic while 58/116 (50\%) candidates were unique to MosaicHunter (Table 1; Additional file 2: Figure S2). Of the 58 candidates unique to MosaicHunter, 35 were filtered out by EM-mosaic on the basis of insufficient alternate allele read support, 16 had a non-zero allelic depth in the parents, and 7 failed quality filters. The 251 candidates unique to EM-mosaic were discarded by the MosaicHunter pipeline during BAM reprocessing $(n=13)$, quality filtering $(n=146)$, and application of LR cutoff (22), or were not called due to inadequate read depth $(n=70)$ (Fig. 1).

\section{Sequence confirmation of candidate mosaic variants}

Candidate mosaic variants from the EM-mosaic and MosaicHunter were combined into a single list for further evaluation. From the 367 high-confidence EMmosaic and/or MosaicHunter mosaic SNVs, we selected 143 candidates (75 uniquely identified by EM-mosaic; 46 uniquely identified by MosaicHunter; 22 identified by both) for experimental confirmation using MiSeq amplicon resequencing (Table 1; Additional file 1: Table S5; Additional file 1: Table S11; Additional file 1: Table S12; "Methods"). DNA fragments encompassing the putative mosaic variant were PCR-amplified from proband and each parent DNA, sequenced on an Illumina MiSeq next-generation sequencer and VAF was calculated for each individual. These candidate mosaics included SNVs on the extremes of the VAF spectrum, as well as mosaics 
that were flagged by MosaicHunter quality filters. In total, we confirmed 108 of 143 candidates as mosaic (Additional file 2: Figure S3A-B), including 21/22 (95\%) sites identified by both pipelines. Candidate variants were considered confirmed by MiSeq analyses if they demonstrated an amplicon VAF exceeding 0.01 but less than 0.45 , so as to indicate a variant of post-zygotic origin. MiSeq VAF values closely correlated with those originally determined by exome sequencing $\left(P=2.2 \times 10^{-16}\right)$. Average MiSeq sequencing read depth was 4639 among all candidates and 4354 among confirmed mosaics.

Based on MiSeq VAF values, we confirmed 85/97 $(88 \%)$ of EM-mosaic candidate mosaic variants with a mean read depth of 4460 (Additional file 2: Figure S3A, C). Three candidate variants were likely germline de novo SNVs $(\mathrm{VAF}>0.45)$. Nine candidate variants were "false positives" that were neither germline de novo SNVs nor mosaic SNVs since either no variant reads were detected by MiSeq sequencing of the proband amplicon, or the same small fraction of variants were detected in proband amplicon and one parent's amplicon.

Parallel analyses with MosaicHunter confirmed 44/68 (65\%) candidate mosaic variants with a mean read depth of 4505 (Additional file 2: Figure S3B,D). There were 23 sites for which no variant reads were detected by MiSeq amplicon sequencing (MiSeq VAF $<0.001$ ) or in which the same small fraction of variant reads was detected in the proband amplicon as in one parent's amplicon.

\section{Mosaic detection power calibrated by sequencing depth and estimated true frequency of mosaicism}

To better characterize how sequencing data parameters affect the detection of mosaic variants, we considered whether estimates of mosaic variant frequency were sensitive to whole exome sequencing depth by calibrating estimates of mosaic detection power using properties of the sequence data (average read depth, prior mosaic fraction, and the value of our overdispersion parameter $\theta$ ) (Additional file 2: Figure S4; Supplemental Methods). Our projected mosaic detection power curves demonstrated more than a doubling of power to detect mosaic variants with VAF 0.2 as sequencing depth increases from $40 \times$ to $80 \times$ (Fig. 2c). Projected mosaic detection power curves for less stringent mosaic cutoffs showed similar increases of power with increasing sequencing depth (Additional file 2: Figure S6).

Next, to estimate the "true" frequency of mosaicism per blood DNA exome, independent of average coverage detection power constraints, we estimated the "true" mosaic count in a VAF range by multiplying the number of mosaics by the inverse of the detection power for each VAF bin. Applying this method to the 184 of 309 high-confidence EM-mosaic variants with $\mathrm{VAF}>0.1$, we estimated the adjusted number of mosaics with VAF >
0.1 to be 361 (Additional file 2: Figure S6A, below). Thus, the true frequency of coding mosaics in the blood $(0.4>\mathrm{VAF}>0.1)$ is 0.14 variants per individual, representing a non-negligible class of mutations with potential contribution to genetic risk for congenital heart disease. The estimated true mosaic frequency does not change significantly when using less stringent mosaic definitions (Additional file 2: Figure S6, below). In sum, after excluding sites failing MiSeq confirmation, we identified 332 blood mosaic variants in $2530 \mathrm{CHD}$ probands (Table 1) or 0.13 mosaic variants per subject with a mean VAF of $0.13 \pm 0.06$. We do not anticipate that doubling the sequencing depth would significantly change this estimate-our estimated true frequency of mosaicism above 10\% allele fraction (assuming full detection power) in the coding region was 0.14 per subject.

\section{Mosaic variants occurred most frequently at $\mathrm{CpG}$ sequences}

The nucleotides surrounding candidate mosaic variant mutations were examined to identify whether any dinucleotide sequences were more likely to be associated with mosaicism. Previous studies demonstrated a strong preference for de novo $\mathrm{C}>\mathrm{T}$ mutations at $\mathrm{CpG}$ dinucleotides compared to other dinucleotides due to the spontaneous deamination of 5-methylcytosine [22, 24]. We asked whether the germline de novo variants observed in CHD probands and the 332 mosaic sites demonstrated a similar sequence preference (Fig. 3; Table 1; Additional file 1: Table S3; Additional file 1: Table S4). Of the 2662 germline de novo mutations identified in 2530 CHD probands, 979 variants (37\% of all variants) involved mutation of the cytosine of a $\mathrm{CpG}$ dinucleotide (Fig. 3a). By contrast, 99 (29\% of all mosaic SNVs) of 332 mosaic SNVs altered the cytosine of a CpG dinucleotide; significantly more than expected by chance (2.2× above expectation; $\left.P=2 \times 10^{-15}\right)$. These observations suggest that somatic de novo mutations were 1.4fold less likely to involve a CpG dinucleotide than germline de novo mutations in $\mathrm{CHD}$ probands $(P=0.01$; Fig. 3b). Even ignoring the high $\mathrm{CpG}$ mutation frequency, cytosines and guanines were $\sim 2$-fold more likely to be mutated than adenines or thymidines both for germline mutations and for mosaic variants. Surprisingly, somatic mutations of $\mathrm{A}>\mathrm{C} / \mathrm{T}>\mathrm{G}$ transversions in ApC dinucleotides were $\sim 2$-fold greater than the corresponding germline mutations $\left(P=5 \times 10^{-8}\right.$; Fig. 3b). Dinucleotide frequencies for mosaic variants detected in cardiac tissue DNA are shown in Fig. 3c.

\section{Detection of mosaic mutations in CHD tissues}

In addition to exome data from blood or saliva samples, a subset of participants also had exome sequencing data available from cardiac tissue. Using EM-mosaic and 


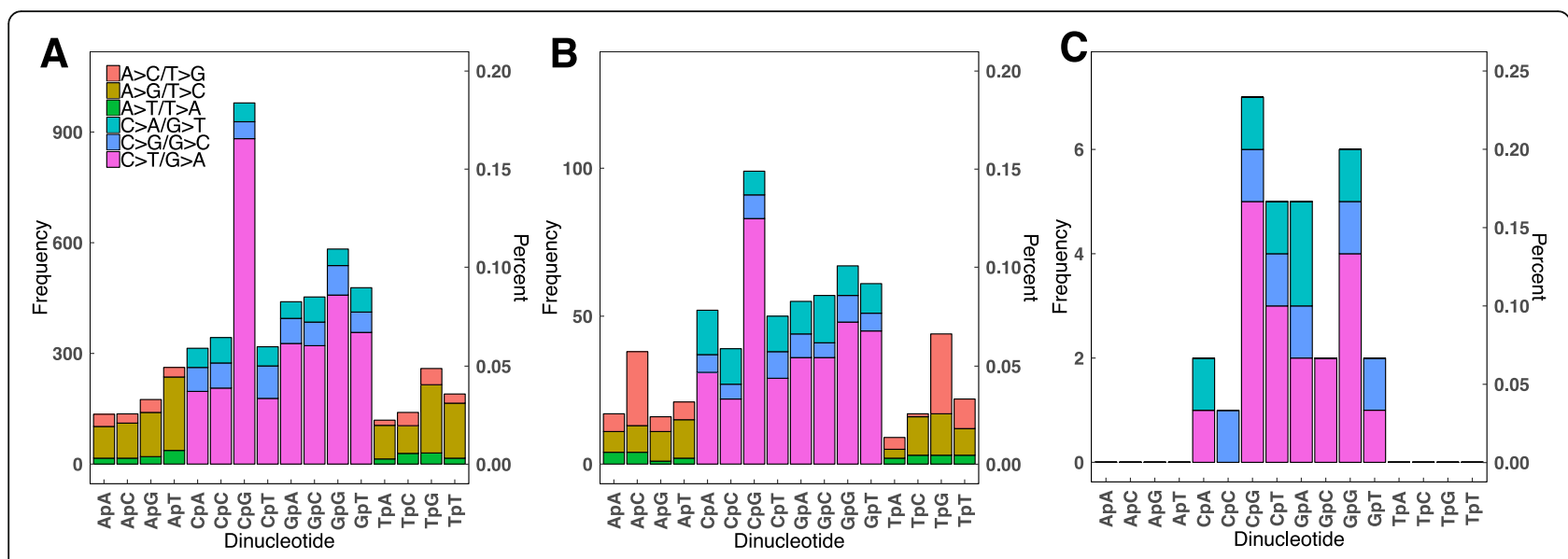

Fig. 3 Mutation spectrum of detected germline and mosaic variants. Rates of specific mutations were compared in a germline, $\mathbf{b}$ blood mosaic, and $\mathbf{c}$ cardiac tissue mosaic variants. Transitions predominated in both variant sets

MosaicHunter, we analyzed exome sequences from 70 cardiac tissues derived from 66 subjects with CHD (Additional file 1: Table S6) and paired blood samples. Among 57 de novo variants (allele fraction approximately 0.5 ) that were previously identified in bloodderived DNA, 54 were also found in CHD tissues. Of the 3 de novo variants not present in cardiac tissue, 1 was outside of the tissue WES capture region and 2 occurred in a single proband (Table 2). In addition, 23 distinct candidate mosaic variants were detected by EM-mosaic
( $n=13)$, MosaicHunter $(n=6)$, or by both algorithms $(n=4)$. We also detected 5 mosaic candidates in blood WES data that had non-zero read depth in the cardiac tissue WES data corresponding to the same individual but below our minimum alternate allele read depth requirement. All 28 candidates identified in either blood or cardiac tissue were tested via MiSeq amplicon sequencing using both blood and cardiac tissue DNA; 15 of 28 (57\%) unique candidate mosaics were confirmed (Table 2; Additional file 1: Table S7), including a CCNC

Table 2 Mosaics detected in individuals with matched cardiovascular tissue and blood

\begin{tabular}{|c|c|c|c|c|c|c|c|c|c|c|}
\hline \multirow[t]{2}{*}{ ID } & \multirow[t]{2}{*}{ Gene } & \multirow{2}{*}{$\begin{array}{l}\text { Variant } \\
\text { class }\end{array}$} & \multirow[t]{2}{*}{ Pipeline } & \multicolumn{4}{|l|}{ CHD tissue } & \multicolumn{3}{|c|}{ Blood WES VAF } \\
\hline & & & & Location & WES AD & WES VAF & MiSeq VAF & WES AD & WES VAF & MiSeq VAF \\
\hline $1-00543$ & CTCFL & Bmis & EM-mosaic & $\mathrm{AO}$ & 138,36 & 0.21 & 0.32 & 29,8 & 0.22 & 0.19 \\
\hline $1-00984$ & ZNF16 & syn & EM-mosaic & LV & 262,1 & 0.00 & 0.01 & 100,7 & 0.07 & 0.07 \\
\hline $1-01282$ & GABRA6 & Dmis & MosaicHunter & RV & 104,1 & 0.01 & 0.01 & 55,12 & 0.18 & 0.18 \\
\hline $1-01684$ & CCNC & Bmis & Both & AoValve, RV & 36,7 & 0.16 & $0.17,0.19$ & 224,40 & 0.15 & 0.14 \\
\hline $1-02672$ & TORTA & syn & Both & AtrSpt & 159,10 & 0.06 & 0.10 & 29,6 & 0.17 & 0.19 \\
\hline $1-03512$ & RFX3 & LoF & MosaicHunter & RV & 156,15 & 0.09 & 0.08 & 39,0 & 0.00 & 0.03 \\
\hline $1-04652$ & PCDH10 & syn & Both & AtrSpt & 154,19 & 0.11 & 0.14 & 15,1 & 0.06 & 0.10 \\
\hline $1-07004$ & ANK2 & Bmis & MosaicHunter & SubAoMembr & 226,13 & 0.05 & 0.04 & 30,0 & 0.00 & 0.00 \\
\hline $1-07004$ & MYH14 & Bmis & Both & SubAoMembr & 124,22 & 0.15 & 0.27 & 33,0 & 0.00 & 0.00 \\
\hline $1-07004$ & $N R G 3$ & Bmis & EM-mosaic & SubAoMembr & 152,30 & 0.16 & 0.24 & 43,0 & 0.00 & 0.00 \\
\hline $1-07004$ & NUDT21 & Bmis & Both & SubAoMembr & 137,22 & 0.14 & 0.14 & 74,0 & 0.00 & 0.02 \\
\hline $1-07004$ & TET3 & Dmis & MosaicHunter & SubAoMembr & 131,1 & 0.01 & 0.03 & 81,16 & 0.16 & 0.27 \\
\hline 1-07299 & RRS1 & syn & Both & RV, UNK & 160,25 & 0.14 & 0.25 & 22,2 & 0.08 & 0.14 \\
\hline 1-09869 & PIK3C2G & LoF & MosaicHunter & LV & 126,9 & 0.07 & 0.10 & 31,0 & 0.00 & 0.00 \\
\hline $1-11800$ & TMEM45A & Bmis & MosaicHunter & RV & 213,0 & 0.00 & 0.00 & 32,7 & 0.18 & 0.06 \\
\hline
\end{tabular}

Characteristics of mosaic variants predicted for individuals with blood and cardiovascular tissue WES data available. Among 15 mosaics, 5 were detected via analysis of blood WES, 8 were detected from cardiovascular tissue WES, and 2 were detected by both approaches. Six of 7 (86\%) mosaics detected from analysis of blood were present in both DNA sources with MiSeq VAF $\geq 0.01$. Two additional variants previously identified as de novo germline variants in blood WES were absent from CHD tissue WES. Minimum 1023 MiSeq reads used to determine VAF. Note: multiple cardiovascular tissue samples were available for participants 101684 and 1-07299. Abbreviations: $A D$ allelic depth (reference, alternate), AO aorta, AtrSpt atrial septum, Bmis benign missense, Dmis deleterious missense, LOF loss of function variant, $L V$ left ventricle, $R V$ right ventricle, $V A F$ variant allele fraction 
variant that was identified in two different cardiac tissues from proband 1-01684 and an RRS1 variant identified in two different cardiac tissues in proband 1-07299. Ten of 15 (66\%) confirmed mosaic variants were detected in blood and cardiac tissues (VAF $>0.01$ ), four were found only in cardiac tissue, and one was found only in blood. Of the 7 mosaics detected by blood WES analysis, 4 were confirmed in the corresponding cardiac tissue sample. Remarkably, five confirmed cardiac tissue mosaic variants occurred in one proband (1-07004), one of which was also present in blood DNA.

These analyses indicate an observed frequency of coding mosaics in the cardiac tissues of 0.23 per individual (15 confirmed mosaics among 66 probands). In order to estimate the true frequency of mosaicism, we applied the same power-based adjustment approach to the 12 of 15 mosaics with allele fraction greater than $10 \%$. Considering the increased sequencing depth $(150 \times)$ in our cardiac tissue samples, our estimated true frequency of coding mosaics with VAF $>0.1$ is 0.21 per individual (14 mosaics among 66 probands; 2 confirmed mosaics with $\mathrm{VAF}>0.1+2$ additional mosaics assuming full detection power). Finally, comparing the allele fraction of mosaics detected in probands with both blood and cardiac tissue available, we found that mosaics with higher VAF in blood were more likely to be found in both tissues (Mann-Whitney $U$ test $P=0.019$ ), presumably indicating that the mutation occurred earlier in lineage development (Fig. 4; Additional file 1: Table S7).

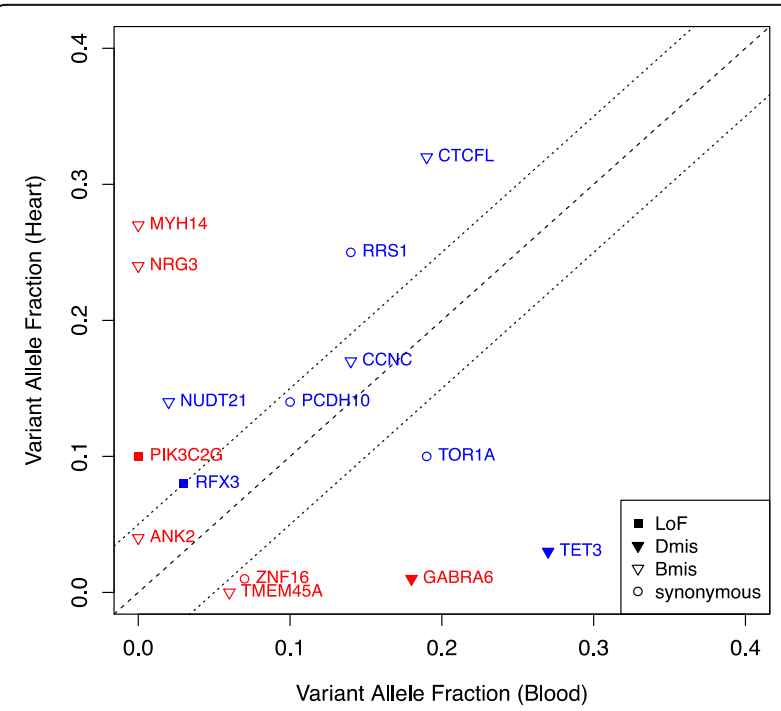

Fig. 4 Validated mosaics detected in probands with matched blood and cardiovascular tissue samples available. Validation VAF from blood compared to validation VAF from cardiovascular tissue demonstrated tissue-specific mosaicism (red) as well as shared mosaicism (blue). Predicted effect of mosaic variants corresponds to marker shape
Blood and cardiac tissue mosaics likely to contribute to CHD Our prior genetic studies of CHD studies showed that damaging de novo variants typically occurred in genes highly expressed in the top quartile of the developing E9.5 mouse heart (HHE) [34, 86] or contributed to CHD in mouse models [42]. Among the 347 mosaic variants identified from blood or cardiac tissue analyses that were not false by MiSeq, 65 altered these HHE and/or mouse CHD genes $(n=4558$; Additional file 1: Table S8). RefSeq functional annotation predicted 52 variants as likely damaging variants (LOF, Dmis), and 46 as likely benign, missense (Additional file 1: Table S8; Additional file 1: Table S9). In total, we observed potentially CHD-causing mosaic mutations in 25 participants, representing $1 \%$ of the 2530 total participants in our CHD cohort. Among these 25 mosaics, we confirmed 22/22 (100\%) candidates tested via MiSeq. Notably, multiple likely damaging mosaic variants altered genes (ISL1, SETD2, NOVA2, SMAD9, LZTR1, KCTD10, KCTD20, $F Z D 5$, and $Q K I$ ) involved in key developmental pathways, which may account for the extracardiac phenotypes observed in these patients (Table 3; Additional file 1: Table S10). There was no difference in the proportion of individuals with extracardiac features among those with damaging mosaic variants compared to the overall cohort (11/ 25 vs $909 / 2521, P=0.68$ ), and there was a wide range of CHD subtypes. Among genes harboring multiple mosaic variants, none carried more than one mosaic mutation predicted to be damaging (Additional file 1: Table S13). Eight genes were found to harbor one damaging (LoF or Dmis) mosaic mutations and at least one damaging germline variant (Additional file 1: Table S14). Three of the eight genes have more than one damaging germline variants. Among these, FBN1 and LZTR1 are well-known risk genes implicated with syndromes that include heart defects. WASHC5 has been implicated with RitscherSchinzel syndrome under a recessive model in an isolated community [18], with CHD as one of the main clinical features. In our cohort, having two damaging germline and one mosaic mutations supports WASHC5 to be a candidate CHD gene. No CNVs were detected in these subjects, with the exception of 1-00192 (duplication at chr15: 22062306-23062355; non-overlapping with the GLYR1 mosaic).

If mosaic variants were unrelated to $\mathrm{CHD}$, we would expect similar allelic fractions between mosaics with variants predicted as likely damaging or likely benign. However, we found that the allele fraction of likely damaging variants in CHD-related genes (union of HHE and mouse CHD genes) was significantly higher (MannWhitney $U$ test $P=0.001$; Fig. 5a). Moreover, among mosaic variants in non-CHD-related genes, we found no significant difference in allele fraction $(P=0.985$; Fig. $5 b)$. We repeated these analyses using (i) less stringent 


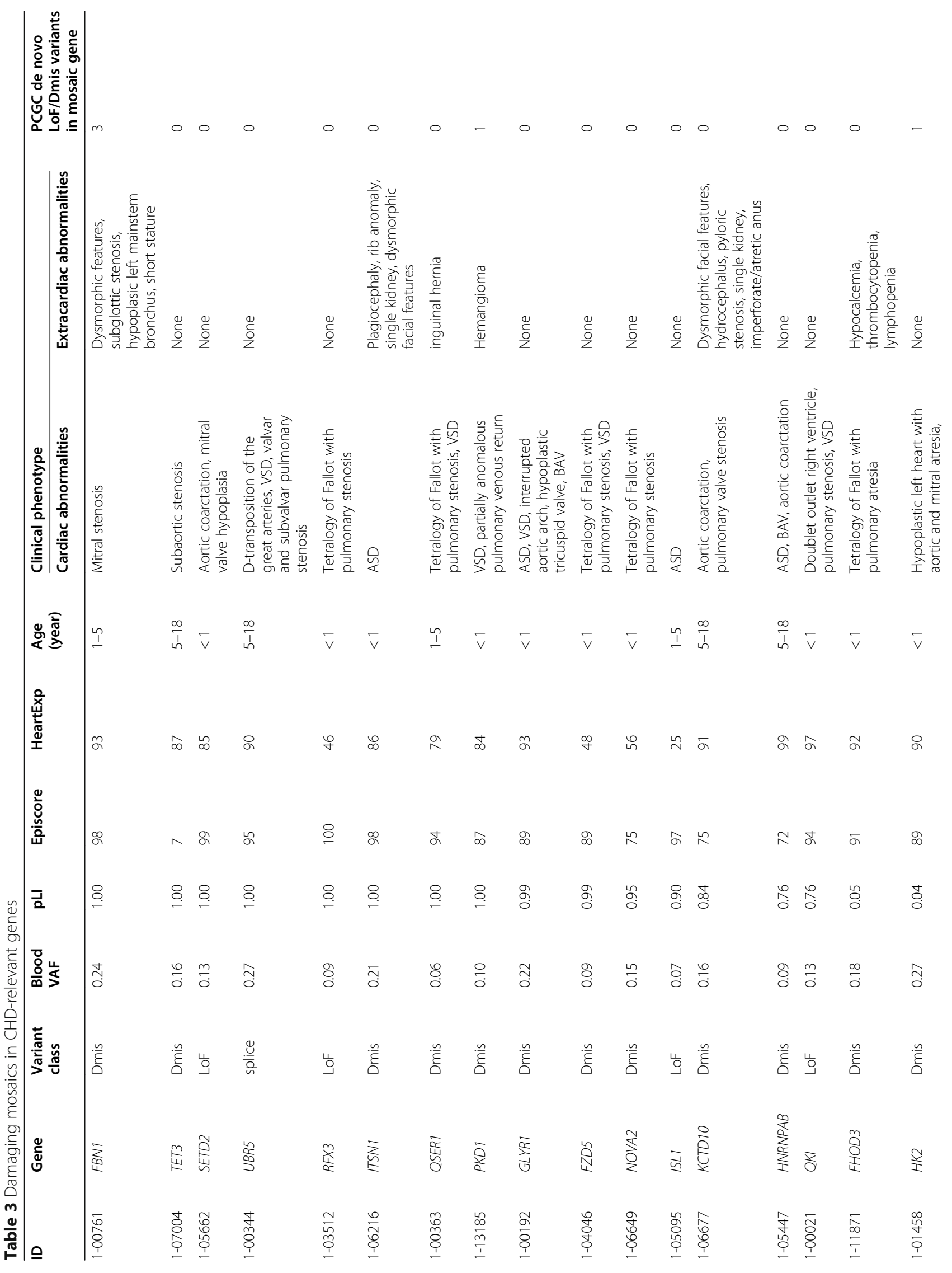




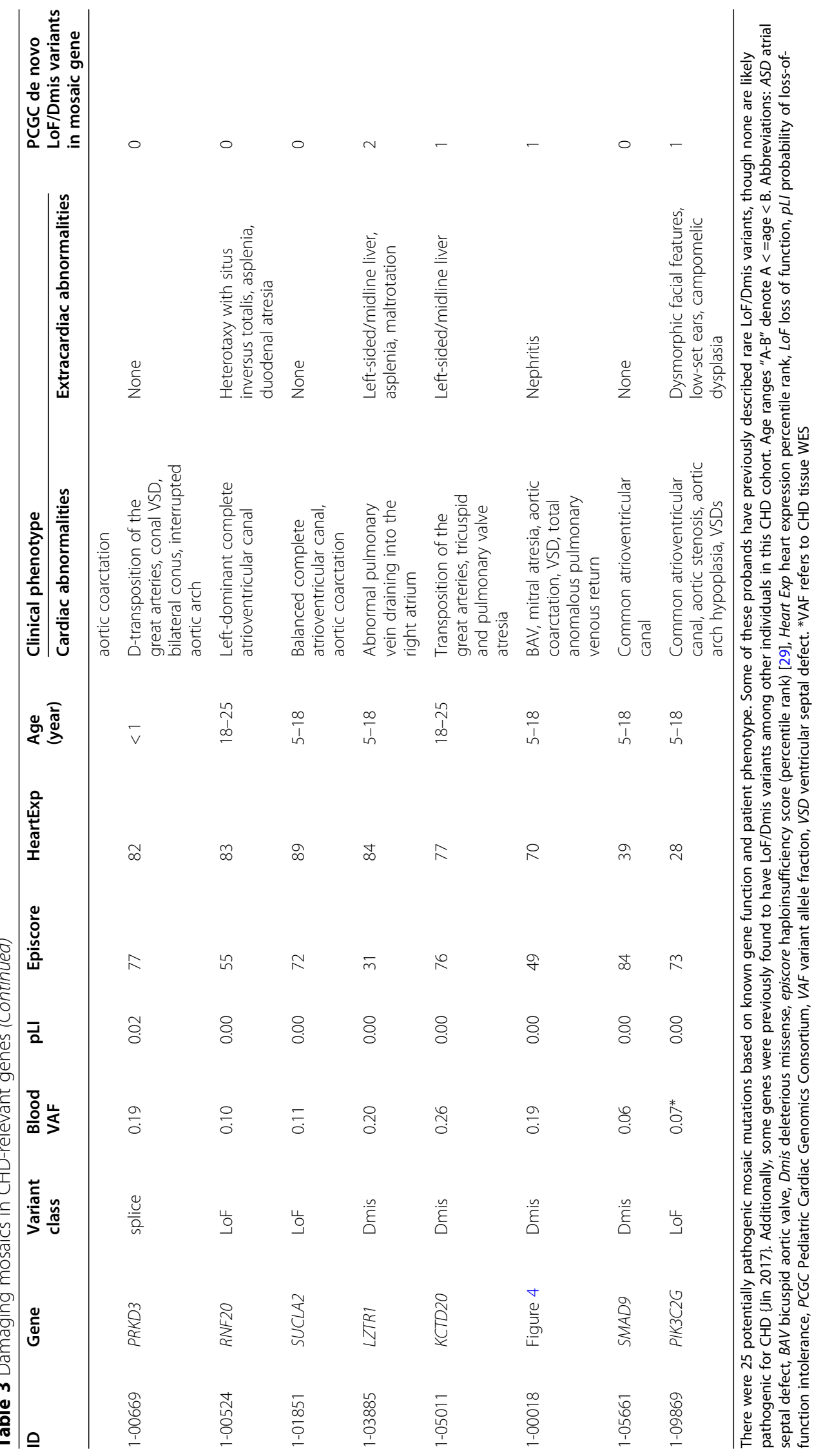



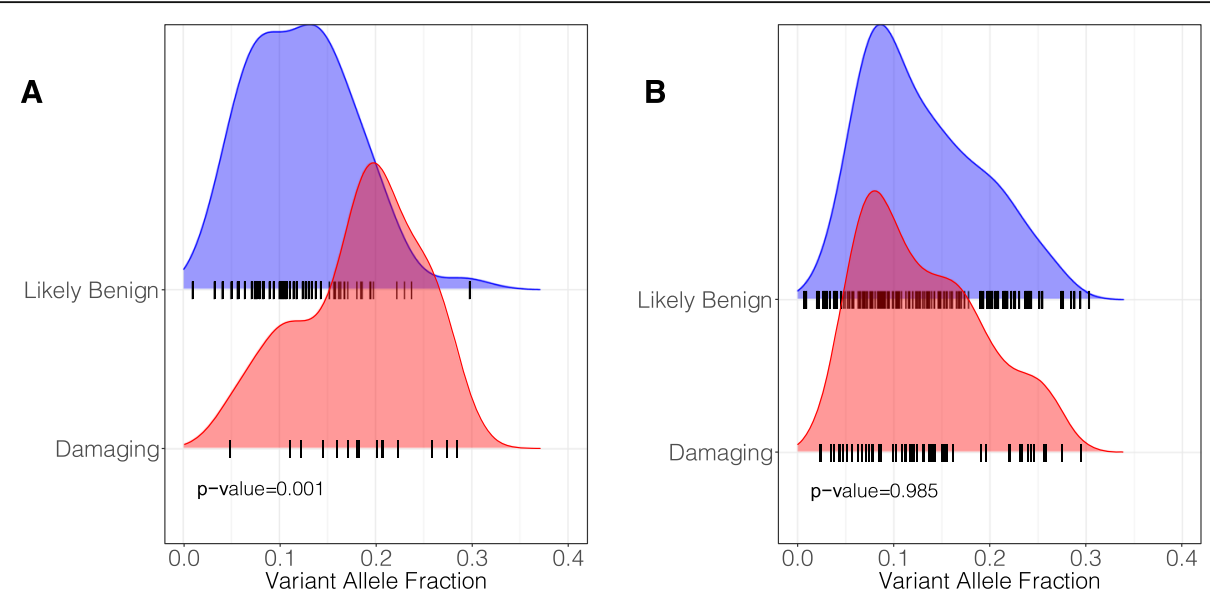

Fig. 5 Damaging mosaics in CHD-related genes have higher variant allele fraction than likely benign mosaics. a Among the 76 mosaics in CHDrelated genes, likely damaging variants have a higher VAF than likely benign (Mann-Whitney U $p=0.001$ ). b Among the 233 mosaics in other (non-CHD-related) genes, there is no difference in VAF based on predicted effect $(p=0.985)$

posterior odds cutoffs of 2 and 5 (Figure S8) and (ii) after excluding the 9 mosaics detected from saliva DNA (Additional file 2: Figure S14) and found the same result. Together, these data support our conclusion that at least some likely damaging mosaic variants identified here contribute to CHD. These results were determined independently of MiSeq validation results.

\section{Discussion}

Distinguishing mosaic mutations from constitutional mutations has both clinical management and reproductive implications for proband and parents. Individuals with mosaic mutations are generally clinically less severely affected for conditions that affect multiple parts of the body $[8,15,19,30,82,84]$. Mutations that occur post-zygotically should have no recurrence risk for the parents and could have a recurrence risk of less than $50 \%$ for the proband depending on gonadal involvement. This study is among the first investigations of the role of post-zygotic mosaic mutations in CHD. We developed a new computational method to robustly detect mosaic single-nucleotide variants from blood WES data at standard read depth. Contrary to existing methods, EMmosaic estimates prior mosaic fraction directly from the data instead of using a fixed parameter, which improves our ability to distinguish high allele fraction mosaic mutations from germline mutations. Additionally, our method also uses a stringent filtering approach to remove false positive calls, minimizing their impact on downstream mosaic detection and improving model specificity. Applying this method to a cohort of $2530 \mathrm{CHD}$ patients, EM-mosaic detected 309 high-confidence mosaics (with a confirmation frequency of $88 \%$ in a subset of variants assessed) or 0.12 variants per proband. Sequencing of cardiac tissue to greater depth identified an additional 8 mosaic variants that had not been detected in blood WES, 6 of which are present in cardiac tissue but not blood. We found more variants per proband in cardiac tissue DNA (0.23 variants per proband) than in blood DNA (0.13 variants per proband). While the increased numbers of mosaic variants in cardiac tissue DNA vs blood DNA may reflect technical (capture method) differences such as sequencing read depth or coverage uniformity of cardiac tissue DNA vs blood DNA, it is possible that somatic variation occurs more frequently in cardiac tissue of CHD probands than in their blood. Ten of 15 mosaic variants among those identified in our 66 CHD proband cardiac tissues had higher VAF in cardiac tissue than in blood (Table 2) and 5 of 15 variants among these individuals had a higher VAF in blood than in tissue.

In total, we observed potentially CHD-causing mosaic mutations in 25 participants, representing $1 \%$ of the 2530 total participants in our CHD cohort. Among these 25 mosaics, we confirmed 22/22 (100\%) candidates tested. We found that in CHD-related genes, likely damaging mosaic mutations have significantly greater alternative allele fraction than likely benign mosaics, suggesting that some of these variants contribute to CHD. Comparison of blood and cardiovascular tissues demonstrated tissue-specific mosaic variants, though those variants with a higher VAF were more likely to be shared between tissues. Due to limitations of conventional clinical interpretation for both mosaic and constitutional CHD variants (Supplemental Methods), we cannot know with complete certainty which among these 25 variants is pathogenic and instead propose that, among our detected mosaics, the 23 detected from blood WES data provide an estimate of the disease-causing mosaics detectable in blood with standard exome 
sequencing read depth. Nine of these variants affect genes known to have a role in cardiac development: ISL1, SETD2, NOVA2, QKI, SMAD9, LZTR1, KCTD10, KCTD20, and FZD5.

The mosaic LoF mutation in ISL1 is likely to be the cause of CHD in participant 1-05095. ISL1 is a transcription factor essential to normal cardiac development that regulates expression of $N K X, G A T A$, and $T B X$ family genes $[9,28]$ and controls secondary heart field differentiation and atrial septation [5, 9]. ISL1 deficiency has been shown to lead to severe CHD in mice $[6,28]$. Participant 1-05095 has an isolated atrial septal defect consistent with a secondary heart field defect phenotype [75] and has no other previously reported damaging germline variants in CHD-related genes.

Damaging germline de novo variants in CHD subjects are enriched in genes related to chromatin modification and RNA processing [34, 42]. Three genes with damaging mosaic variants discovered here have related functions. SETD2 is a histone methyltransferase required for embryonic vascular remodeling [36]; it is both sensitive to haploinsufficiency and highly expressed in the heart during development. NOVA2 is a key alternative-splicing regulator involved in angiogenesis that has been shown to disrupt vascular lumen formation when depleted [27]. QKI encodes an RNA-binding protein that regulates splicing, RNA export from the nucleus, protein translation, and RNA stability [48]. QKI is also highly expressed in the heart during development and has been shown to cause CHD and other blood vessel defects in mice when dysregulated [62].

Other damaging mosaic variants affect processes known to be relevant to CHD. SMAD9 is involved in the TGF-beta signaling pathway. TGF-beta signaling plays a critical role in cardiac development and cardiovascular physiology, leading to pulmonary arterial hypertension and cardiac abnormalities in mice when dysregulated [17, 74]. LZTR1 encodes a member of the BTB-Kelch superfamily that is highly expressed in the heart during development and has been associated with Noonan [26, 85] and DiGeorge Syndromes [46], both of which are characterized by CHD. The individual with the LZTR1 damaging mosaic variant did have pulmonary lymphangiectasias that are a less common feature of Noonan syndrome, but did not have other clinical findings common to Noonan syndrome. KCTD10 binds to and represses the transcriptional activity of TBX5 (T-box transcription factor), which plays a dose-dependent role in the formation of cardiac chambers [80]. KCTD10 is highly expressed in the heart during development and has been shown to produce CHD in mice when dysregulated [67]. KCTD2O is a positive regulator of $A k t$ [61] also highly expressed in the heart during development. FZD5 is haploinsufficient and encodes a transmembrane receptor involved in Wnt, mTOR, and Hippo signaling pathways and has been shown to play a role in cardiac development [10]. The individual with a damaging mosaic variants in $F B N 1$, which is associated with several genetic syndromes, had features consistent with WeillMarchesani syndrome such as brachycephaly, mitral valve stenosis, short stature, and midface hypoplasia.

Finally, two mosaic variants found in cardiac tissue, genes encoding RFX3 and PIK3C2G, may be diseaserelevant. PIK3C2G is a signaling kinase involved in cell proliferation, survival, and migration, as well as oncogenic transformation and protein trafficking (OMIM: 609001). The effects of PIK3C2G haploinsufficiency during cardiac development have not been characterized. RFX3 is a highly constrained ciliogenic transcription factor that leads to pronounced laterality defects [65], and disruption of RFX3 leads to congenital heart malformations in mice (MGI: 5560494) [72]. Notably, the RFX3 LoF variant has a fourfold higher VAF in cardiac tissue than in blood.

Three capture platforms were used in this study. The main technical difference between capture platforms is depth of coverage. We do not believe this to confound the main results of this study. Regarding (1) the estimated rate of mosaic mutations in coding regions-we estimated the rate based on observed number of mosaic mutations normalized by the detection power of such mutations in individual samples. The sequencing depth was a main factor considered in our calculation of detection power. Regarding (2) the genetic contribution of mosaic mutations to $\mathrm{CHD}$-we concluded that mosaic mutations contribute to CHD based on the fact that the damaging mosaic mutations have overall greater allele fraction than benign mosaic mutations in plausible CHD genes. Since capture method and sequencing depth are independent of the type of variants in each gene, we do not expect capture kit to confound this analysis.

Several investigators, who studied cancer and diseases with cutaneous manifestations, proposed that the VAF correlates with time of mutation acquisition and disease burden [3, 31, 71]. In this study, we used VAF as a proxy for cellular percentage and mutational timing, with increasing VAF corresponding to events occurring earlier in development. Thus, we assume that CHD-associated mosaic events identified in blood-derived DNA occurred during or shortly after the gastrulation process (third week of development) [60] in the mesodermal progenitor cells that differentiate into both heart precursor cells (cardiogenic mesoderm) and blood precursor cells (hemangioblasts). We found that in CHD-relevant genes, mosaic sites predicted to be damaging tended to have higher VAF than sites predicted to be likely benign, consistent with the hypothesis that these mutations arose early in fetal development and play significant roles in 
CHD. However, additional functional studies are necessary to fully assess causality.

We recognize that while our method is able to detect a large fraction of mosaic variants in blood, our calibrated estimates for the true number of mosaics suggest there are a non-negligible number of additional mutations that were not identified by our method. At our current average sequencing depth of $60 \times$, we have limited sensitivity in the low VAF $(<0.05)$ range. To reliably identify these low allelic fraction sites, ultra-deep sequencing will be critical to distinguishing true variants from noise. At $500 \times$, we estimate detection sensitivity for mosaic events at VAF 0.05 to be above $80 \%$. Additionally, copy number variations (CNVs) are well documented as contributors to CHD [87] and somatic CNVs comprise a class of potentially impactful mosaic events. However, neither of the methods presented are designed to detect mosaic $\mathrm{CNVs}$ since the computational problem of detecting mosaic CNVs is very different to detecting mosaic SNV/indels. We also recognize age-related clonal hematopoiesis $[25,40]$ as a potential confounding factor in somatic mutation detection; however, our study cohort includes mostly pediatric cases and we did not observe mosaic mutations in genes related to clonal expansion (e.g., ASXL1, DNMT3A, TET2, JAK2) nor did we observe a relationship between proband age and mosaic rate (Additional file 2: Figure S9), suggesting minimal impact from this process. We also did not find evidence of a relationship between parental age (paternal or maternal) and proband mosaicism (Additional file 1: Table S16; Additional file 2: Figure S10; Additional file 2: Figure S11).

In this manuscript, we presented the results of our case-only analysis due to the lack of appropriate controls. To allow direct comparison, controls would need to be matched on the basis of age, sex, sequencing depth, and DNA source (blood). While at the time of this study such controls were not available, recent efforts to promote data sharing and availability may yield an appropriate set of controls in the near future and enable estimating the contribution of mosaicism to $\mathrm{CHD}$ with higher resolution and certainty.

\section{Conclusions}

This study is among the first investigations of the role of post-zygotic mosaic mutations in CHD. Despite limitations in sequencing depth and sample type, EM-mosaic was able to detect 309 high-confidence mosaics from blood, with resequencing confirmation in $88 \%$ of cases assessed, and 17 candidates in cardiac tissue (41\% confirmation rate). Using MosaicHunter, an additional 64 candidate mosaic sites were identified, of which 23/46 (50\%) candidates from blood DNA and 4/6 (67\%) from CHD tissue DNA validated. We observed mosaic frequencies of 0.13/individual in blood and 0.23/individual in cardiac tissue. Assuming full detection power, we estimate the true frequency of mosaic variants in the coding region above $10 \%$ mosaicism to be 0.14 /individual in blood and 0.26/individual in cardiac tissue. In total, we observed potentially CHD-causing mosaic mutations in 25 participants, representing $1 \%$ of our $\mathrm{CHD}$ cohort, and propose that these 25 cases provide an estimate of the disease-causing mosaics detectable in blood with standard exome sequencing read depth. Additionally, we found that in CHD-related genes, likely damaging mosaics have significantly greater alternative allele fraction than likely benign mosaics, suggesting that many of these variants cause CHD and occurred early in development. In the subset of our cohort for which cardiovascular tissue samples were available, we show that mosaics detected in blood can also be found in the diseaserelevant tissue and that, while the VAF for mosaic variants often differed between blood and cardiovascular tissue DNA, variants with higher VAF were more likely to be shared between tissues. Given current limitations in sequencing depth and on the availability of relevant tissues, particularly for conditions impacting internal organs like the heart, the full extent of the role of mosaicism in many diseases remains to be explored. However, as datasets containing larger numbers of blood and other tissue samples sequenced at higher depths become increasingly available, we will be able to more fully characterize the biological processes underlying postzygotic mutation and, by extension, the contribution of mosaicism to disease using the methods presented here.

\section{Additional files}

Additional file 1. Contains Supplemental Tables S1-S16.

Additional file 2. Contains Supplemental Figures S1-S14.

Additional file 3. Contains Supplemental Methods.

\begin{abstract}
Abbreviations
ASD: Autism spectrum disorder; CHD: Congenital heart disease; dnSNV: De novo SNV; Dmis: Deleterious missense mutation; DP site: Total read depth at a variant site; $\mathrm{DP}_{\text {sample: }}$ Sample-wide average read depth; ExAC: Exome Aggregation Consortium; FDR: False discovery rate; gnomAD: Genome Aggregation Database; HHE: High heart expression; LOF: Loss of function; LR: Likelihood ratio; MAF: Minor allele frequency; N: Total read depth; $N_{\text {alt }}$ : Alternate allele read depth; OR: Odds ratio; PCGC: Pediatric Cardiac Genomics Consortium; pLI: Probability of loss-of-function intolerance; PV4: P value for strand bias, baseQ bias, mapQ bias and tail distance bias (SAMtools); SNV: Single-nucleotide variant; VAF: Variant allele frequency; WES: Whole exome sequencing
\end{abstract}

\section{Acknowledgements}

The authors would like to thank all the participants and their families.

\section{Authors' contributions}

YS, JGS, CES, and WKC conceived and oversaw the study. AH, SUM, JALW, $H Q, K B M, J G S, C E S, Y S$, and WKC analyzed the data. AH developed the EMmosaic pipeline and wrote the statistical analysis code. SUM and JALW carried out MosaicHunter analyses of blood and tissue samples. JMG, AT, and SD performed MiSeq experimental confirmation. AH, SUM, EG, CES, and WKC 
interpreted the impact of mosaics on participant clinical phenotypes. DB, RWK, JWN, GAP, DS, MT-F, MB, RPL, EG, BDG, CES, JGS, and WKC were involved in cohort ascertainment, phenotypic characterization, and recruitment. DM collected cardiovascular tissue samples. AH, SUM, JALW, YS, JGS, CES, and WKC wrote the manuscript. All authors read and approved the manuscript.

\section{Funding}

This work was supported by the National Heart, Lung, and Blood Institute (NHLBI) grants for the Pediatric Cardiac Genomics Consortium [U01HL098188, U01-HL131003, UM1-HL098147, U01-HL098153, U01-HL098163, UM1-HL098123, UM1-HL098162, UM1-HL128761, UM1-HL128711], National Institute of General Medical Sciences (R01GM120609), National Library of Medicine training grant (T15LM007079), and Columbia University CTSA Precision Medicine Training Fellowship (TL1TR001875).

\section{Availability of data and materials}

EM-mosaic and custom scripts and pipeline for analyzing data are available from https://github.com/ShenLab/mosaicism [34]. The MosaicHunter software is available from http://mosaichunter.cbi.pku.edu.cn/ [36]. SAMtools is available from http://www.htslib.org/ [52]. ANNOVAR is available from http://annovar. openbioinformatics.org/en/latest/ [85]. Integrative Genomics Viewer (IGV) software is available from https://software.broadinstitute.org/software/igv/ [70]. Whole exome sequencing data have been deposited in the database of Genotypes and Phenotypes (dbGaP) under accession numbers phs000571.v1.p1 [11], phs000571.v2.p1 [12], and phs000571.v3.p2 [13].

\section{Ethics approval and consent to participate}

CHD subjects were recruited to the Congenital Heart Disease Network Study of the Pediatric Cardiac Genomics Consortium (CHD GENES: ClinicalTrials.gov identifier NCT01196182). The institutional review boards of Boston's Children's Hospital (protocol number 10-08-0404), Brigham and Women's Hospital (protocol number 2010P002667/PHS), Great Ormond Street Hospital (protocol number REC reference: 10/H0711/98; IRAS project ID: 34974), Children's Hospital of Los Angeles (protocol number CCl-10-00245), Children's Hospital of Philadelphia (protocol number 10-007730), Columbia University Medical Center (protocol number AAAF3161), Icahn School of Medicine at Mount Sinai (protocol number HS\#: 10-00616; GCO\#1: 09-0104(0001) NHLBI/ $\mathrm{NIH})$, Rochester School of Medicine and Dentistry (protocol number RSRB00033081), Steven and Alexandra Cohen Children's Medical Center of New York (protocol number 10-258A), and Yale School of Medicine (protocol number 1010007481) approved the protocols. All subjects or their parents provided informed consent. Our research conformed to the Declaration of Helsinki.

\section{Consent for publication}

Not applicable.

\section{Competing interests}

The authors declare that they have no competing interests.

\section{Author details}

${ }^{1}$ Columbia University Medical Center, 1130 St Nicholas Ave, New York, NY 10032, USA. ${ }^{2}$ Boston Children's Hospital, Boston, MA, USA. ${ }^{3}$ Harvard Medical School, Boston, MA, USA. ${ }^{4}$ Icahn School of Medicine at Mount Sinai, New York, NY, USA. ${ }^{5}$ Stanford University, Palo Alto, CA, USA. ${ }^{6}$ Children's Hospital Los Angeles, Los Angeles, CA, USA. 'University of Rochester Medical Center, Rochester, NY, USA. ${ }^{8}$ Gladstone Institutes and University of California San Francisco, San Francisco, CA, USA. ' $U$ niversity of Utah School of Medicine, Salt Lake City, UT, USA. ${ }^{10}$ Yale University School of Medicine, New Haven, CT, USA. ${ }^{11}$ Rockefeller University, New York, NY, USA. ${ }^{12}$ Children's Hospital of Philadelphia, Philadelphia, PA, USA. ${ }^{13}$ Brigham and Women's Hospital, Boston, MA, USA. ${ }^{14}$ Howard Hughes Medical Institute, Harvard University, Boston, MA, USA.

Received: 9 September 2019 Accepted: 9 April 2020 Published online: 29 April 2020

\section{References}

1. Acuna-Hidalgo $R$, Bo T, Kwint MP, van de Vorst M, Pinelli M, Veltman JA, et al. Post-zygotic point mutations are an Underrecognized source of de novo genomic variation. Am J Hum Genet. 2015;97(1):67-74. https://doi.org/ 10.1016/J.AJHG.2015.05.008.

2. Auton A, Abecasis GR, Altshuler DM, Durbin RM, Bentley DR, Chakravarti A, et al. A global reference for human genetic variation. Nature. 2015;526:6874. https://doi.org/10.1038/nature15393.

3. Belickova M, Vesela J, Jonasova A, Pejsova B, Votavova H, Merkerova MD, et al. TP53 mutation variant allele frequency is a potential predictor for clinical outcome of patients with lower-risk myelodysplastic syndromes. Oncotarget. 2016;7(24):36266-79. https://doi.org/10.18632/oncotarget.9200.

4. Biesecker $L G$, Spinner NB. A genomic view of mosaicism and human disease. Nat Rev Genet. 2013;14(5):307-20. https://doi.org/10.1038/nrg3424.

5. Briggs LE, Kakarla J, Wessels $A$. The pathogenesis of atrial and atrioventricular septal defects with special emphasis on the role of the dorsal mesenchymal protrusion. Differentiation. 2012;84(1):117-30. https:// doi.org/10.1016/j.diff.2012.05.006

6. Cai C-L, Liang X, Shi Y, Chu P-H, Pfaff SL, Chen J, Evans S. Isl1 identifies a cardiac progenitor population that proliferates prior to differentiation and contributes a majority of cells to the heart. Dev Cell. 2003;5(6):877-89. Retrieved from http://www.ncbi.n/m.nih.gov/pubmed/14667410.

7. Cibulskis K, Lawrence MS, Carter SL, Sivachenko A, Jaffe D, Sougnez C, et al. Sensitive detection of somatic point mutations in impure and heterogeneous cancer samples. Nat Biotechnol. 2013;31(3):213-9. https:// doi.org/10.1038/nbt.2514.

8. Cohn DH, Starman BJ, Blumberg B, Byers PH. Recurrence of lethal osteogenesis imperfecta due to parental mosaicism for a dominant mutation in a human type I collagen gene (COL1A1). Am J Hum Genet. 1990;46(3):591-601. Retrieved from http://www.ncbi.nlm.nih.gov/ pubmed/2309707.

9. Colombo S, de Sena-Tomás C, George V, Werdich AA, Kapur S, MacRae CA, Targoff KL. nkx genes establish SHF cardiomyocyte progenitors at the arterial pole and pattern the venous pole through Isl1 repression. Dev. 2017: 161497. https://doi.org/10.1242/dev.161497.

10. Dawson K, Aflaki M, Nattel S. Role of the Wnt-frizzled system in cardiac pathophysiology: a rapidly developing, poorly understood area with enormous potential. J Physiol. 2013;591(6):1409-32. https://doi.org/10.1113/ jphysiol.2012.235382.

11. dbGaP/database of Genotypes and Phenotypes/ National Center for Biotechnology Information, National Library of Medicine (NCBI/NLM)/https:// www.ncbi.nlm.nih.gov/gap/; accession phs000571.v1.p1 [https://www.ncbi. nlm.nih.gov/projects/gap/cgi-bin/study.cgi?study_id=phs000571.v1.p1].

12. dbGaP/database of Genotypes and Phenotypes/ National Center for Biotechnology Information, National Library of Medicine (NCBI/NLM)/https:// www.ncbi.nlm.nih.gov/gap/; accession phs000571.v2.p1 [https://www.ncbi. nlm.nih.gov/projects/gap/cgi-bin/study.cgi?study_id=phs000571.v2.p1].

13. dbGaP/database of Genotypes and Phenotypes/ National Center for Biotechnology Information, National Library of Medicine (NCBI/NLM)/https:// www.ncbi.nlm.nih.gov/gap/; accession phs000571.v3.p2 [https://www.ncbi. nlm.nih.gov/projects/gap/cgi-bin/study.cgi?study_id=phs000571.v3.p2].

14. DePristo MA, Banks E, Poplin R, Garimella KV, Maguire JR, Hartl C, et al. A framework for variation discovery and genotyping using next-generation DNA sequencing data. Nat Genet. 2011;43(5):491-8. https://doi.org/10.1038/ng.806.

15. Donkervoort S, Hu Y, Stojkovic T, Voermans NC, Foley AR, Leach ME, et al. Mosaicism for dominant collagen 6 mutations as a cause for intrafamilial phenotypic variability. Hum Mutat. 2015;36(1):48-56. https://doi.org/10.1002/ humu.22691.

16. Dou Y, Yang $X$, Li Z, Wang S, Zhang Z, Ye AY, et al. Postzygotic singlenucleotide mosaicisms contribute to the etiology of autism spectrum disorder and autistic traits and the origin of mutations. Hum Mutat. 2017; 38(8):1002-13. https://doi.org/10.1002/humu.23255.

17. Drake KM, Comhair SA, Erzurum SC, Tuder RM, Aldred MA. Endothelial chromosome 13 deletion in congenital heart disease-associated pulmonary arterial hypertension dysregulates SMAD9 signaling. Am J Respir Crit Care Med. 2015;191(7):850-4.

18. Elliott AM, Simard LR, Coghlan G, Chudley AE, Chodirker BN, Greenberg CR, et al. A novel mutation in KIAA0196: identification of a gene involved in Ritscher-Schinzel/3C syndrome in a first nations cohort. J Med Genet. 2013; 50(12):819-22. https://doi.org/10.1136/jmedgenet-2013-101715.

19. Etheridge SP, Bowles NE, Arrington CB, Pilcher T, Rope A, Wilde AAM, et al. Somatic mosaicism contributes to phenotypic variation in Timothy syndrome. Am J Med Genet A. 2011;155(10):2578-83. https://doi.org/10. 1002/ajmg.a.34223. 
20. Finger JH, Smith CM, Hayamizu TF, McCright IJ, Xu J, Law M, et al. The mouse gene expression database (GXD): 2017 update. Nucleic Acids Res. 2017;45(D1):D730-6. https://doi.org/10.1093/nar/gkw1073.

21. Fischbach GD, Lord C. The Simons simplex collection: a resource for identification of autism genetic risk factors. Neuron. 2010;68(2):192-5. https://doi.org/10.1016/j.neuron.2010.10.006.

22. Francioli LC, Polak PP, Koren A, Menelaou A, Chun S, Renkens I, et al. Genome-wide patterns and properties of de novo mutations in humans. Nat Genet. 2015;47(7):822-6. https://doi.org/10.1038/ng.3292.

23. Freed D, Pevsner J. The contribution of mosaic variants to autism Spectrum disorder. PLoS Genet. 2016;12(9):e1006245. https://doi.org/10.1371/journal. pgen. 1006245.

24. Fryxell KJ, Moon W-J. CpG mutation rates in the human genome are highly dependent on local GC content. Mol Biol Evol. 2005;22(3):650-8. https://doi. org/10.1093/molbev/msi043

25. Genovese G, Kähler AK, Handsaker RE, Lindberg J, Rose SA, Bakhoum SF, et al. Clonal hematopoiesis and blood-cancer risk inferred from blood DNA sequence. N Engl J Med. 2014;371(26):2477-87. https://doi.org/10.1056/ NEJMoa1409405

26. Ghedira N, Kraoua L, Lagarde A, Abdelaziz RB, Olschwang S, Desvignes JP, et al. Further evidence for the implication of LZTR1, a gene not associated with the Ras-Mapk pathway, in the pathogenesis of Noonan syndrome. Biol Med. 2017;09(06):4-7. https://doi.org/10.4172/0974-8369.1000414.

27. Giampietro C, Deflorian G, Gallo S, Di Matteo A, Pradella D, Bonomi S, et al The alternative splicing factor Nova2 regulates vascular development and lumen formation. Nat Commun. 2015;6:1-15. https://doi.org/10.1038/ ncomms 9479 .

28. Golzio C, Havis E, Daubas P, Nuel G, Babarit C, Munnich A, et al. ISL1 directly regulates FGF10 transcription during human cardiac outflow formation. PLoS One. 2012;7(1):e30677. https://doi.org/10.1371/journal.pone.0030677.

29. Han X, Chen S, Flynn E, Wu S, Wintner D, Shen Y. Distinct epigenomic patterns are associated with haploinsufficiency and predict risk genes of developmental disorders. Nature Communications. 2018;9(1):2138. https:// doi.org/10.1038/s41467-018-04552-7.

30. Happle R. The McCune-Albright syndrome: a lethal gene surviving by mosaicism. Clin Genet. 1986;29(4):321-4. Retrieved from http://www.ncbi. nlm.nih.gov/pubmed/3720010.

31. Happle R. Mosaicism in human skin. Understanding the patterns and mechanisms. Arch Dermatol. 1993;129(11):1460-70. Retrieved from http:// www.ncbi.n/m.nih.gov/pubmed/8239703.

32. Haeussler M, Zweig AS, Tyner C, Speir ML, Rosenbloom KR, Raney BJ, et al. The UCSC genome browser database: 2019 update. Nucleic Acids Res. 2019; 47(D1):D853-8. https://doi.org/10.1093/nar/gky1095.

33. Heinrich V, Stange J, Dickhaus T, Imkeller P, Krüger U, Bauer S, et al. The allele distribution in next-generation sequencing data sets is accurately described as the result of a stochastic branching process. Nucleic Acids Res. 2012;40(6):2426-31. https://doi.org/10.1093/nar/gkr1073.

34. Homsy J, Zaidi S, Shen Y, Ware JS, Samocha KE, Karczewski KJ, et al. De novo mutations in congenital heart disease with neurodevelopmental and other congenital anomalies. Science. 2015;350(6265):1262-6. https://doi.org/ 10.1126/science.aac9396.

35. Hsieh A, Morton SU, Willcox JAL, Gorham JM, Tai AC, Qi H, et al. Early postzygotic mutations contribute to congenital heart disease. BioRxiv. 2019;733105 https://doi.org/10.1101/733105 [https://github.com/ShenLab/mosaicism].

36. Hu M, Sun X-J, Zhang Y-L, Kuang Y, Hu C-Q, Wu W-L, et al. Histone H3 lysine 36 methyltransferase Hypb/Setd2 is required for embryonic vascular remodeling. Proc Natl Acad Sci. 2010;107(7):2956-61. https://doi.org/10. 1073/pnas.0915033107.

37. Huang AY, Zhang Z, Ye AY, Dou Y, Yan L, Yang X, et al. MosaicHunter: accurate detection of postzygotic single-nucleotide mosaicism through next-generation sequencing of unpaired, trio, and paired samples. Nucleic Acids Res. 2017;45(10):e76. https://doi.org/10.1093/nar/gkx024. [http:// mosaichunter.cbi.pku.edu.cn/].

38. Ioannidis NM, Rothstein JH, Pejaver V, Middha S, McDonnell SK, Baheti S, et al. REVEL: an ensemble method for predicting the pathogenicity of rare missense variants. Am J Hum Genet. 2016;99(4):877-85. https://doi.org/10. 1016/j.ajhg.2016.08.016.

39. Jaganathan K, Kyriazopoulou Panagiotopoulou S, McRae JF, Darbandi SF, Knowles D, Li Yl, et al. Predicting splicing from primary sequence with deep learning. Cell. 2019;176(3):535-548.e24. https://doi.org/10.1016/J.CELL.2018. 12.015 .
40. Jaiswal S, Fontanillas P, Flannick J, Manning A, Grauman PV, Mar BG, et al. Age-related clonal hematopoiesis associated with adverse outcomes. N Eng J Med. 2014;371(26):2488-98. https://doi.org/10.1056/NEJMoa1408617.

41. Jamuar SS, Lam A-TN, Kircher M, D'Gama AM, Wang J, Barry BJ, et al. Somatic mutations in cerebral cortical malformations. N Engl J Med. 2014; 371(8):733-43. https://doi.org/10.1056/NEJMoa1314432.

42. Jin SC, Homsy J, Zaidi S, Lu Q, Morton S, DePalma SR, et al. Contribution of rare inherited and de novo variants in 2,871 congenital heart disease probands. Nat Genet. 2017;49(11):1593-601. https://doi.org/10.1038/ng.3970.

43. Karczewski KJ, Weisburd B, Thomas B, Solomonson M, Ruderfer DM, Kavanagh D, et al. The ExAC browser: displaying reference data information from over 60000 exomes. Nucleic Acids Res. 2017;45(D1):D840-5. https:// doi.org/10.1093/nar/gkw971.

44. Karczewski KJ, Francioli LC, Tiao G, Cummings BB, Alföldi J, Wang Q, et al. Variation across 141,456 human exomes and genomes reveals the spectrum of loss-of-function intolerance across human protein-coding genes. BioRxiv. 2019:531210 https://doi.org/10.1101/531210.

45. Krupp DR, Barnard RA, Duffourd Y, Evans SA, Mulqueen RM, Bernier R, et al. Exonic mosaic mutations contribute risk for autism spectrum disorder. Am J Hum Genet. 2017;101(3):369-90. https://doi.org/10.1016/j.ajhg.2017.07.016.

46. Kurahashi H, Akagi K, Inazawa J, Ohta T, Niikawa N, Kayatani F, et al. Isolation and characterization of a novel gene deleted in DiGeorge syndrome. Hum Mol Genet. 1995;4(4):541-9. Retrieved from http://www.ncbi.nlm.nih.gov/ pubmed/7633402

47. Kurek KC, Luks VL, Ayturk UM, Alomari Al, Fishman SJ, Spencer SA, et al. Somatic mosaic activating mutations in PIK3CA cause CLOVES syndrome. Am J Hum Genet. 2012;90(6):1108-15. https://doi.org/10.1016/j.ajhg.2012.05. 006.

48. Lauriat TL, Shiue L, Haroutunian V, Verbitsky M, Ares M, Ospina L, Mclnnes $L A$. Developmental expression profile ofquaking, a candidate gene for schizophrenia, and its target genes in human prefrontal cortex and hippocampus shows regional specificity. J Neurosci Res. 2008;86(4):785-96. https://doi.org/10.1002/jnr.21534.

49. Lee JH, Huynh M, Silhavy JL, Kim S, Dixon-Salazar T, Heiberg A, et al. De novo somatic mutations in components of the PI3K-AKT3-mTOR pathway cause hemimegalencephaly. Nat Genet. 2012;44(8):941-5. https://doi.org/10. 1038/ng.2329.

50. Lek M, Karczewski KJ, Minikel EV, Samocha KE, Banks E, Fennell T, et al. Analysis of protein-coding genetic variation in 60,706 humans. Nature. 2016; 536(7616):285-91. https://doi.org/10.1038/nature19057.

51. Li, H. (2013). Aligning sequence reads, clone sequences and assembly contigs with BWA-MEM. Retrieved from http://arxiv.org/abs/1303.3997.

52. Li H. A statistical framework for SNP calling, mutation discovery, association mapping and population genetical parameter estimation from sequencing data. Bioinformatics (Oxford, England). 2011;27(21):2987-93. https://doi.org/ 10.1093/bioinformatics/btr509. [http://www.htslib.org/]

53. Li H. Toward better understanding of artifacts in variant calling from highcoverage samples. Bioinformatics (Oxford, England). 2014;30(20):2843-51. https://doi.org/10.1093/bioinformatics/btu356.

54. Lim ET, Uddin M, De Rubeis S, Chan Y, Kamumbu AS, Zhang X, et al. Rates, distribution and implications of postzygotic mosaic mutations in autism spectrum disorder. Nat Neurosci. 2017;20(9):1217-24. https://doi.org/10. 1038/nn.4598.

55. Lindhurst MJ, Parker VER, Payne F, Sapp JC, Rudge S, Harris J, et al. Mosaic overgrowth with fibroadipose hyperplasia is caused by somatic activating mutations in PIK3CA. Nat Genet. 2012;44(8):928-33. https://doi.org/10.1038/ ng.2332.

56. Liu X, Wu C, Li C, Boerwinkle E. dbNSFP v3.0: a one-stop database of functional predictions and annotations for human nonsynonymous and splice-site SNVs. Hum Mutat. 2016:37(3):235-41. https://doi.org/10.1002/humu.22932.

57. Manheimer KB, Richter F, Edelmann L, D'Souza SL, Shi L, Shen Y, et al. Robust identification of mosaic variants in congenital heart disease. Hum Genet. 2018;137(2):183-93. https://doi.org/10.1007/s00439-018-1871-6.

58. McKenna A, Hanna M, Banks E, Sivachenko A, Cibulskis K, Kernytsky A, et al. The genome analysis toolkit: a MapReduce framework for analyzing nextgeneration DNA sequencing data. Genome Res. 2010;20(9):1297-303. https://doi.org/10.1101/gr.107524.110.

59. McDonald J, Wooderchak-Donahue WL, Henderson K, Paul E, Morris A, Bayrak-Toydemir P. Tissue-specific mosaicism in hereditary hemorrhagic telangiectasia: implications for genetic testing in families. Am J Med Genet A. 2018;176(7):1618-21. https://doi.org/10.1002/ajmg.a.38695. 
60. Moorman A, Webb S, Brown NA, Lamers W, Anderson RH. Development of the heart: (1) formation of the cardiac chambers and arterial trunks. Heart (British Cardiac Society). 2003;89(7):806-14. Retrieved from http://www.ncbi. nlm.nih.gov/pubmed/12807866.

61. Nawa M, Matsuoka M. KCTD20, a relative of BTBD10, is a positive regulator of Akt. BMC Biochem. 2013;14(1):27. https://doi.org/10.1186/1471-2091-14-27.

62. Noveroske JK, Lai L, Gaussin V, Northrop JL, Nakamura H, Hirschi KK, Justice MJ. Quaking is essential for blood vessel development. Genesis (New York, N.Y. : 2000). 2002;32(3):218-30. Retrieved from http://www.ncbi.nlm.nih.gov/ pubmed/11892011.

63. O'Leary NA, Wright MW, Brister JR, Ciufo S, Haddad D, McVeigh R, et al. Reference sequence (RefSeq) database at NCBI: current status, taxonomic expansion, and functional annotation. Nucleic Acids Res. 2016;44(D1):D73345. https://doi.org/10.1093/nar/gkv1189.

64. Poduri A, Evrony GD, Cai X, Elhosary PC, Beroukhim R, Lehtinen MK, et al. Somatic activation of AKT3 causes hemispheric developmental brain malformations. Neuron. 2012;74(1):41-8. https://doi.org/10.1016/j.neuron. 2012.03.010

65. Ramsdell AF. Left-right asymmetry and congenital cardiac defects: getting to the heart of the matter in vertebrate left-right axis determination. Dev Biol. 2005;288(1):1-20. https://doi.org/10.1016/J.YeDBIO.2005.07.038.

66. Ramu A, Noordam MJ, Schwartz RS, Wuster A, Hurles ME, Cartwright RA, Conrad DF. DeNovoGear: de novo indel and point mutation discovery and phasing. Nat Methods. 2013;10(10):985-7. https://doi.org/10.1038/nmeth.2611.

67. Ren K, Yuan J, Yang M, Gao X, Ding X, Zhou J, et al. KCTD10 is involved in the cardiovascular system and notch signaling during early embryonic development. PLoS One. 2014;9(11):e112275. https://doi.org/10.1371/journal. pone.0112275

68. Rentzsch P, Witten D, Cooper GM, Shendure J, Kircher M. CADD: predicting the deleteriousness of variants throughout the human genome. Nucleic Acids Res. 2019;47(D1):D886-94. https://doi.org/10.1093/nar/gky1016.

69. Rivière J-B, Mirzaa GM, O'Roak BJ, Beddaoui M, Alcantara D, Conway RL, et al. De novo germline and postzygotic mutations in AKT3, PIK3R2 and PIK3CA cause a spectrum of related megalencephaly syndromes. Nat Genet. 2012;44(8):934-40. https://doi.org/10.1038/ng.2331.

70. Robinson JT, Thorvaldsdóttir H, Winckler W, Guttman M, Lander ES, Getz G, Mesirov JP. Integrative genomics viewer. Nat Biotechnol. 2011;29:24-6. https://doi.org/10.1038/nbt.1754 [https://software.broadinstitute.org/ software/igv/]

71. Sallman DA, Komrokji R, Vaupel C, Cluzeau T, Geyer SM, McGraw KL, et al. Impact of TP53 mutation variant allele frequency on phenotype and outcomes in myelodysplastic syndromes. Leukemia. 2016;30(3):666-73. https://doi.org/10.1038/leu.2015.304.

72. Smith CL, Blake JA, Kadin JA, Richardson JE, Bult CJ, Mouse Genome Database Group. Mouse genome database (MGD)-2018: knowledgebase for the laboratory mouse. Nucleic Acids Res. 2018;46(D1):D836-42. https://doi. org/10.1093/nar/gkx1006

73. Smith KS, Yadav VK, Pei S, Pollyea DA, Jordan CT, De S. SomVarlUS: somatic variant identification from unpaired tissue samples. Bioinformatics. 2016; 32(6):808-13. https://doi.org/10.1093/bioinformatics/btv685.

74. Soubrier F, Chung WK, Machado R, Grünig E, Aldred M, Geraci M, et al Genetics and genomics of pulmonary arterial hypertension. J Am Coll Cardiol. 2013;62(25):D13-21. https://doi.org/10.1016/J.JACC.2013.10.035.

75. Stevens KN, Hakonarson H, Kim CE, Doevendans PA, Koeleman BPC, Mital S, et al. Common variation in ISL1 confers genetic susceptibility for human congenital heart disease. PLoS One. 2010;5(5):e10855. https://doi.org/10. 1371/journal.pone.0010855.

76. Stosser MB, Lindy AS, Butler E, Retterer K, Piccirillo-Stosser CM, Richard G, McKnight DA. High frequency of mosaic pathogenic variants in genes causing epilepsy-related neurodevelopmental disorders. Genet Med. 2018; 20(4):403-10. https://doi.org/10.1038/gim.2017.114.

77. Sun JX, He Y, Sanford E, Montesion M, Frampton GM, Vignot S, et al. A computational approach to distinguish somatic vs. germline origin of genomic alterations from deep sequencing of cancer specimens without a matched normal. PLoS Comput Biol. 2018;14(2):e1005965. https://doi.org/10. 1371/journal.pcbi.1005965.

78. Symoens S, Steyaert W, Demuynck L, De Paepe A, Diderich KEM, Malfait F, Coucke PJ. Tissue-specific mosaicism for a lethal osteogenesis imperfecta COL1A1 mutation causes mild OI/EDS overlap syndrome. Am J Med Genet A. 2017:173(4):1047-50. https://doi.org/10.1002/ajmg.a.38135.
79. Tate JG, Bamford S, Jubb HC, Sondka Z, Beare DM, Bindal N, et al. COSMIC: the catalogue of somatic mutations in cancer. Nucleic Acids Res. 2019; 47(D1):D941-7. https://doi.org/10.1093/nar/gky1015.

80. Tong $X, Z u$ Y, Li Z, Li W, Ying L, Yang J, et al. Kctd10 regulates heart morphogenesis by repressing the transcriptional activity of Tbx5a in zebrafish. Nat Commun. 2014;5:1-10. https://doi.org/10.1038/ncomms4153.

81. Van der Auwera GA, Carneiro MO, Hartl C, Poplin R, del Angel G, LevyMoonshine A, et al. From fastQ data to high-confidence variant calls: the genome analysis toolkit best practices pipeline. Curr Protoc Bioinform. 2013; (SUPL.43) https://doi.org/10.1002/0471250953.bi1110s43.

82. Wallis GA, Starman BJ, Zinn AB, Byers PH. Variable expression of osteogenesis imperfecta in a nuclear family is explained by somatic mosaicism for a lethal point mutation in the alpha 1(I) gene (COL1A1) of type I collagen in a parent. Am J Hum Genet. 1990;46(6):1034-40. Retrieved from http://www.ncbi.n/m.nih.gov/pubmed/2339700.

83. Wang K, Li M, Hakonarson H. ANNOVAR: functional annotation of genetic variants from high-throughput sequencing data. Nucleic Acids Res. 2010; 38(16) https://doi.org/10.1093/nar/gkq603 [http://annovar. openbioinformatics.org/en/latest/].

84. Weinstein MM, Kang T, Lachman RS, Bamshad M, Nickerson DA, Krakow D, Cohn DH. Somatic mosaicism for a lethal TRPV4 mutation results in nonlethal metatropic dysplasia. Am J Med Genet A. 2016;170(12):3298-302. https://doi.org/10.1002/ajmg.a.37942.

85. Yamamoto GL, Aguena M, Gos M, Hung C, Pilch J, Fahiminiya S, et al. Rare variants in SOS2 and LZTR1 are associated with Noonan syndrome. J Med Genet. 2015;52(6):413-21. https://doi.org/10.1136/jmedgenet-2015-103018.

86. Zaidi S, Brueckner M. Genetics and genomics of congenital heart disease. Circ Res. 2017;120(6):923-40. https://doi.org/10.1161/CIRCRESAHA.116. 309140.

87. Zaidi S, Choi M, Wakimoto H, Ma L, Jiang J, Overton JD, et al. De novo mutations in histone-modifying genes in congenital heart disease. Nature. 2013;498(7453):220-3. https://doi.org/10.1038/nature12141.

\section{Publisher's Note}

Springer Nature remains neutral with regard to jurisdictional claims in published maps and institutional affiliations.

Ready to submit your research? Choose BMC and benefit from:

- fast, convenient online submission

- thorough peer review by experienced researchers in your field

- rapid publication on acceptance

- support for research data, including large and complex data types

- gold Open Access which fosters wider collaboration and increased citations

- maximum visibility for your research: over $100 \mathrm{M}$ website views per year

At BMC, research is always in progress.

Learn more biomedcentral.com/submissions 